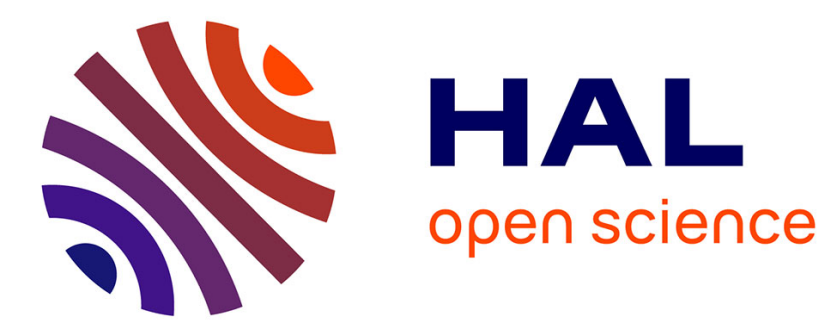

\title{
Response analysis of a laminar premixed M-flame to flow perturbations using a linearized compressible Navier-Stokes solver
}

\author{
Mathieu Blanchard, Thierry Schuller, D. Sipp, P. J. Schmid
}

\section{To cite this version:}

Mathieu Blanchard, Thierry Schuller, D. Sipp, P. J. Schmid. Response analysis of a laminar premixed M-flame to flow perturbations using a linearized compressible Navier-Stokes solver. Physics of Fluids, 2015, 27 (4), pp.043602. 10.1063/1.4918672 . hal-01178888

HAL Id: hal-01178888

https: / hal-polytechnique.archives-ouvertes.fr/hal-01178888

Submitted on 21 Jul 2015

HAL is a multi-disciplinary open access archive for the deposit and dissemination of scientific research documents, whether they are published or not. The documents may come from teaching and research institutions in France or abroad, or from public or private research centers.
L'archive ouverte pluridisciplinaire HAL, est destinée au dépôt et à la diffusion de documents scientifiques de niveau recherche, publiés ou non, émanant des établissements d'enseignement et de recherche français ou étrangers, des laboratoires publics ou privés. 


\section{AIP | Physics of}

\section{Response analysis of a laminar premixed M-flame to flow perturbations using a linearized compressible Navier-Stokes solver}

M. Blanchard, T. Schuller, D. Sipp, and P. J. Schmid

Citation: Physics of Fluids 27, 043602 (2015); doi: 10.1063/1.4918672

View online: http://dx.doi.org/10.1063/1.4918672

View Table of Contents: http://scitation.aip.org/content/aip/journal/pof2/27/4?ver=pdfcov

Published by the AIP Publishing

\section{Articles you may be interested in}

Interactions between turbulence and flames in premixed reacting flows

Phys. Fluids 23, 125111 (2011); 10.1063/1.3671736

A regime diagram for premixed flame kernel-vortex interactions

Phys. Fluids 19, 043604 (2007); 10.1063/1.2720595

On molecular transport effects in real gas laminar diffusion flames at large pressure

Phys. Fluids 17, 103601 (2005); 10.1063/1.1990198

Direct numerical simulation of transitional and turbulent buoyant planar jet flames

Phys. Fluids 16, 4443 (2004); 10.1063/1.1804974

Flame-vortex interaction in a reacting vortex ring

Phys. Fluids 10, 189 (1998); 10.1063/1.869560

\section{Did your publisher get}

18 MILLION DOWNLOADS in 2014?

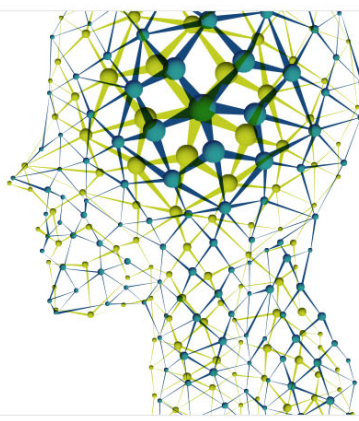




\title{
Response analysis of a laminar premixed M-flame to flow perturbations using a linearized compressible Navier-Stokes solver
}

\author{
M. Blanchard, ${ }^{1, a)}$ T. Schuller, ${ }^{2,3}$ D. Sipp, ${ }^{4}$ and P. J. Schmid ${ }^{5}$ \\ ${ }^{1}$ LadHyX, CNRS and Ecole Polytechnique, 91128 Palaiseau, France \\ ${ }^{2}$ CNRS, UPR 288, Laboratoire d'Energétique Moléculaire et Macroscopique Combustion \\ (EM2C), Grande Voie des Vignes, 92290 Châtenay-Malabry, France \\ ${ }^{3}$ Centrale-Supélec, Grande Voie des Vignes, 92290 Châtenay-Malabry, France \\ ${ }^{4}$ ONERA-DAFE, 8 rue des Vertugadins, 92190 Meudon, France \\ ${ }^{5}$ Department of Mathematics, Imperial College London, London SW7 2AZ, United Kingdom
}

(Received 22 October 2014; accepted 6 April 2015; published online 29 April 2015)

\begin{abstract}
The response of a laminar premixed methane-air flame subjected to flow perturbations around a steady state is examined experimentally and using a linearized compressible Navier-Stokes solver with a one-step chemistry mechanism to describe combustion. The unperturbed flame takes an M-shape stabilized both by a central bluff body and by the external rim of a cylindrical nozzle. This base flow is computed by a nonlinear direct simulation of the steady reacting flow, and the flame topology is shown to qualitatively correspond to experiments conducted under comparable conditions. The flame is then subjected to acoustic disturbances produced at different locations in the numerical domain, and its response is examined using the linearized solver. This linear numerical model then allows the componentwise investigation of the effects of flow disturbances on unsteady combustion and the feedback from the flame on the unsteady flow field. It is shown that a wrinkled reaction layer produces hydrodynamic disturbances in the fresh reactant flow field that superimpose on the acoustic field. This phenomenon, observed in several experiments, is fully interpreted here. The additional perturbations convected by the mean flow stem from the feedback of the perturbed flame sheet dynamics onto the flow field by a mechanism similar to that of a perturbed vortex sheet. The different regimes where this mechanism prevails are investigated by examining the phase and group velocities of flow disturbances along an axis oriented along the main direction of the flow in the fresh reactant flow field. It is shown that this mechanism dominates the low-frequency response of the wrinkled shape taken by the flame and, in particular, that it fully determines the dynamics of the flame tip from where the bulk of noise is radiated. (C) 2015 AIP Publishing LLC. [http://dx.doi.org/10.1063/1.4918672]
\end{abstract}

\section{INTRODUCTION}

This study is motivated by a general lack of understanding of the flame response to flow perturbations. This response is needed to determine the stability of combustors with respect to thermoacoustic instabilities and to predict combustion noise emission from these systems. ${ }^{1}$ These two issues cause several difficulties in the development of high-performance combustion systems, especially as improved efficiency and lower pollutant emission levels are concerned. ${ }^{2,3}$ Gaining insight into the fundamental mechanisms that dominate the response of flames to unsteadiness is mandatory to reducing combustion noise emission and self-sustained combustion instabilities in real systems.

There are different ways to analyze the response of flames to flow perturbations which are briefly reviewed below. An alternative methodology is proposed in this work where the reacting base flow is calculated by direct simulations of the compressible Navier-Stokes, energy, and species

\footnotetext{
a)Electronic mail: mathieu.blanchard@ladhyx.polytechnique.fr
} 
transport equations. The response of this flow to small perturbations is then computed by solving the linearized set of equations around this base state. This linearized solver allows the rapid determination of flame frequency and impulse responses to flow disturbances over the full frequency range of interest and the componentwise investigation of different contributions yielding large unsteady heat release rate fluctuations or maximum sound radiation. This approach offers a general framework for flow sensitivity analysis and optimal forcing.

Before proceeding, it is worth analyzing the current strategies used to infer the response of flames to flow perturbations. In realistic systems with complex geometries, many techniques have been developed to determine the flame frequency response to harmonic flow excitations. Acoustic characterizations are preferred when optical access is limited, as is often the case in real systems. ${ }^{4,5}$ When optical access is possible, this response is generally characterized experimentally by determining flame transfer functions (FTFs) or, more recently, flame describing functions relating heat release rate disturbances to flow rate or mixture composition oscillations produced at some location in the injector for different flow and excitation conditions. ${ }^{6-12}$ These characterizations have proven valuable for linear and nonlinear stability analyses of the system dynamics; in addition, they are used to determine stability charts as the operating conditions of the combustor are modified. ${ }^{5,13-17}$

With the rapid development of high-performance computing resources, it is nowadays also possible to capture the response of flames to flow disturbances in complex combustor geometries by solving the compressible Navier-Stokes equations in multi-species reacting flows using Large-Eddy Simulation (LES) tools. ${ }^{18-20}$ These simulations, however, remain difficult and incur considerable computational costs. For this reason, they are often limited to the analysis of the system response to a single forcing frequency or to the simulation of the dynamics of self-sustained oscillations initiated from small or finite-level initial perturbations to a limit cycle. ${ }^{21-23}$ System identification tools have also been applied to LES simulations to estimate the flame transfer function. ${ }^{24,25}$ These techniques are based on the forcing of the flame using a small-amplitude broadband noise, assuming the flame response to be linear. There is thus an ongoing effort to develop low-order numerical and analytical models aiming at improving the description of the flame dynamics in configurations of increasing complexities at reduced computational effort.

In the case of premixed flames, these low-order models generally rely on a level-set description of the flame front motion that is reduced to a flame sheet separating the fresh reactants from the burnt gases. This interface propagates in the normal direction with the flame speed and is also advected by the flow. Assuming simplified flow and flame topologies and prescribing different types of flow perturbations, it is possible in many cases to describe wrinkles produced along the flame sheet and deduce the resulting heat release rate disturbances. Analytical expressions for flame transfer functions and, more recently, flame impulse responses were determined using this level-set description in different generic laminar and turbulent flow configurations. ${ }^{26-32}$ These models highlight the different physical mechanisms influencing the flame response to the excitation and yield the appropriate dimensionless groups to analyze the response of flames featuring different flow topologies and subjected to different types of perturbations. They are of undeniable value to guide experimental characterizations in practical systems and to suggest control strategies.

There are, however, several difficulties to extend this level-set description to real systems characterized by complex flow topologies and complex interactions of the unsteady flame with the combustion chamber boundaries. The base flow and the flame shape are generally difficult to describe with analytical models in real systems. The way perturbations are produced and transported by or propagating through the flow is also complex to model, in particular, when these disturbances interact with shear layers. ${ }^{33-37}$ One explored possibility has been to couple the level-set description of the flame reaction sheet with the Navier-Stokes equations and then compute the response of this flow to excitations. ${ }^{38-41}$ This type of approach, relying on direct simulations of the Navier-Stokes equations, only differs by the way combustion is modeled. More detailed combustion models are now commonly used to simulate the response of flames to flow forcing. ${ }^{42-45}$

A different approach is undertaken in this work, where only the steady, reacting base flow is solved by direct simulation of the nonlinear Navier-Stokes equations assuming that chemistry proceeds through one irreversible reaction. The unsteady response of the flame is subsequently determined by solving a linearized version of these equations around this base state. This type of approach 
is well-known and widely used in sensitivity analyses of non-reacting flows, ${ }^{46}$ but has only recently been applied to reactive flows. ${ }^{47,48}$ This strategy also differs from the acoustic perturbation equation formulation that has been developed to analyze combustion noise. ${ }^{49}$ In this hybrid approach, the noise sources are extracted from large-eddy simulations and the sound field is computed with an aeroacoustic solver, but the flame does not respond to the incident sound field. In the linear method presented herein, a steady base flame is computed and perturbations are supposed to develop around this base state without modifying it. The methodology is validated in a generic laminar configuration by comparison with experiments. It is then used to improve our understanding of the interactions taking place between the unsteady flow and the flame in response to imposed excitations.

It is thus worth synthesizing the current state of knowledge of unsteady flames and flowinteractions leading to heat release rate disturbances. Only perturbation scales much larger than the chemical-reaction and thermal thicknesses are considered, such that the reaction layer where combustion takes place can legitimately be approximated by an infinitely thin interface separating the reactants from the combustion products. ${ }^{50,51}$ This approximation is valid because flames are known to act as low-pass filters for flow perturbations with a cutoff frequency much lower than frequencies associated with disturbances interfering with the flame's internal structure. ${ }^{52}$ Most studies have considered the canonical problem of flame wrinkling produced by acoustic, hydrodynamic, or mixturecomposition perturbations. For flame sheets inclined with respect to the main direction of the flow, it has been shown that flame wrinkles are produced by disturbances normal to the reaction layer which are convected by the mean flow along the flame front. ${ }^{53}$ The flame motion corresponds then to a delayed system consisting of the convection of flame-root oscillations as well as flow disturbances integrated along the flame front. ${ }^{28,30}$ These wrinkles originate from all conceivable mechanisms altering the flame displacement speed or the velocity field. The physics associated with these different contributions is currently well described, and many effects can be captured by low-order models.

However, the interactions of flame wrinkles with the flow field are still an active field of research. The effects of gas expansion through the flame have been considered in the context of flame stability ${ }^{54,55}$ These efforts led to an accurate description of the Landau-Darrieus instability through linear ${ }^{56}$ and nonlinear ${ }^{57,58}$ analyses. When the system is stable, it is known from experiments that acoustically perturbed flames or flames in a steady flow subjected to oscillations of its anchoring points produce hydrodynamic disturbances in the vicinity of the reaction layer. ${ }^{34,59}$ These disturbances have first been detected in velocity measurements along the symmetry axis of conical flames subjected to acoustic excitations. ${ }^{60}$ Three regimes have been identified. ${ }^{34}$ At low frequencies, the phase lag of velocity fluctuations recorded in the fresh stream of reactants scales with the inverse of the injection flow velocity. At high frequencies, these disturbances propagate in the entire fresh stream at the speed of sound. At intermediate frequencies, transitions between these two regimes can be detected, with abrupt phase changes. The region where flow disturbances are convected narrows closer to the flame front as the forcing frequency increases. This type of feedback has also been identified for flames subjected to mixture-composition oscillations. ${ }^{40,61}$ However, the detailed mechanisms by which the flame modifies the upstream, perturbed flow field remain to be determined and modeled. These issues constitute the second objective of the present work.

The article is organized as follows. Results of experiments conducted for a laminar premixed methane/air M-flame subjected to acoustic forcing are presented in Sec. II. The numerical methodology developed to capture the base flow is then presented in Sec. III together with the simulations of the steady configuration. This section ends with the description of the linear solver. Results for the flame impulse and flame transfer functions are then compared to measurements in Sec. IV. This comparison is used to validate the developed methodology and to identify the flow regions where large heat release rate fluctuations and noise emissions take place. Effects of the flame dynamics on the unsteady flow field are examined in Sec. V.

\section{REFERENCE EXPERIMENTS}

The configuration studied in this work is a laminar M-flame (MF) stabilized at the nozzle outlet of a burner equipped with a central rod (MF in Fig. 1). A methane-air mixture is injected at the 


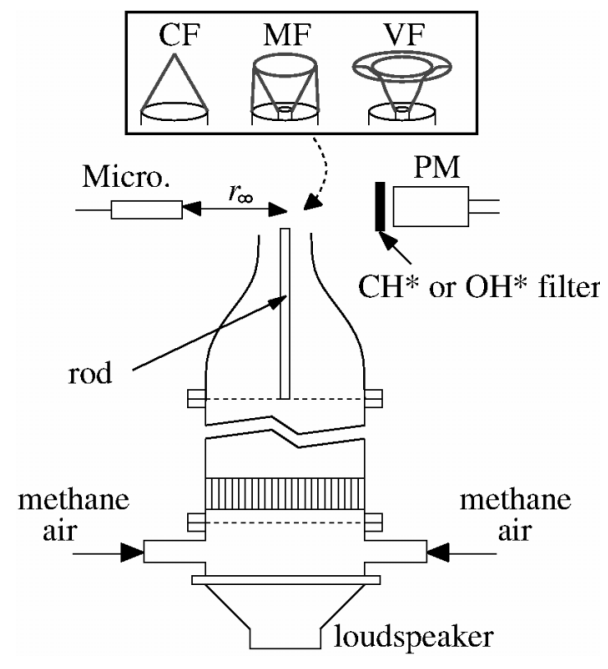

FIG. 1. Experimental setup comprising a laminar burner with a central rod and a loudspeaker to modulate the flow. A PM with interferometric filters $\left(\mathrm{OH}^{*}\right.$ or $\left.\mathrm{CH}^{*}\right)$ collects the light emission from the flame. A microphone (M) records the radiated pressure at a distance $r_{\infty}$ from the burner axis. A LDV and an ICCD, not shown here, are also placed around the setup to measure the flow velocity and to record flame images.

bottom of the burner. This mixture passes successively through a perforated plate, a laminarization honeycomb grid, and a converging nozzle of $r_{1}=11 \mathrm{~mm}$ outlet radius. A cylindrical, metallic rod of $r_{0}=3 \mathrm{~mm}$ radius is placed on the burner axis. The flat top of this rod lies $2 \mathrm{~mm}$ above the nozzle outlet plane. This burner is used to anchor laminar flames featuring different shapes. ${ }^{6,62}$ A conical, $\mathrm{V}$-, or M-flame can be stabilized depending on the injection and ignition conditions (Fig. 2). The case of a flame featuring an M-shape anchored both at the nozzle outlet rim and on the central rod is studied herein. This configuration has been selected because it is very sensitive to flow unsteadiness with large heat release rate fluctuations and large emission of noise. ${ }^{6,62}$

An image of a nearly stoichiometric M-flame, with an equivalence ratio of $\phi=1.04$, is shown in Fig. 2 for a bulk injection velocity $u_{b}=1.97 \mathrm{~m} \mathrm{~s}^{-1}$ at the annular nozzle outlet at room temperature. The flame height measured along the burner axis is $h=16 \mathrm{~mm}$. In the absence of external forcing, the broadband noise level measured in the lab with a microphone set at $r_{\infty}=0.25 \mathrm{~m}$ away from the burner axis does not exceed $70 \mathrm{~dB}$. A loudspeaker placed at the bottom of the burner and shown in Fig. 1 is used to modulate the flow with harmonic excitations. The axial-velocity perturbation produced at the burner outlet is measured by a Laser Doppler Velocimeter (LDV) $z=2 \mathrm{~mm}$ above the injection plane, i.e., at the same level as the top of the central rod and close to the center of the annular rim, $r=7 \mathrm{~mm}$ away from the burner axis. Images of the flame response were recorded with an intensified CCD (ICCD) camera equipped with a UV Nikkor objective that was triggered by the forcing signal sent to the loudspeaker.
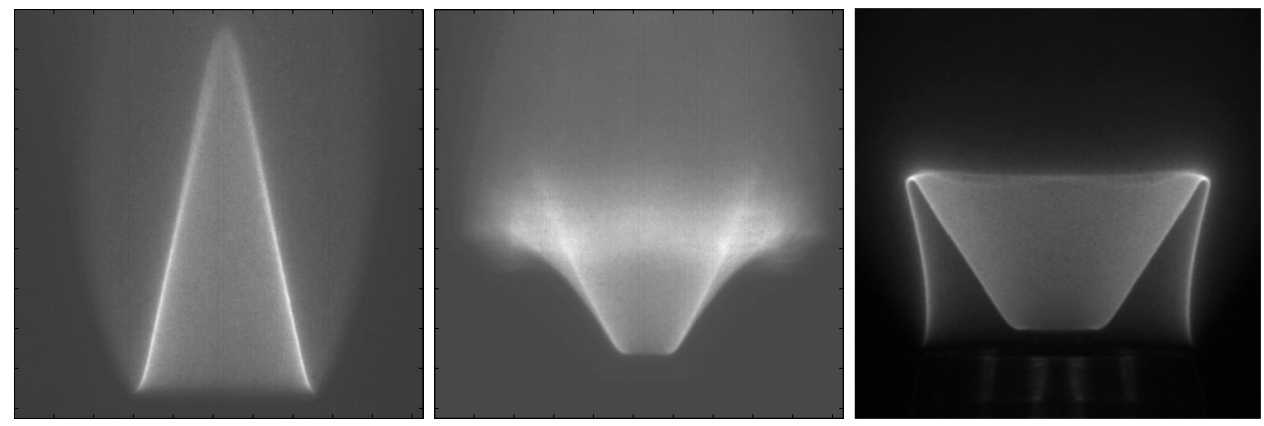

FIG. 2. Flame topologies for different injection and ignition conditions. Left: conical flame, $\phi=1.11, u_{b}=1.7 \mathrm{~m} \mathrm{~s}^{-1}$. Middle: V-flame, $\phi=1.11, u_{b}=2.3 \mathrm{~m} \mathrm{~s}^{-1}$. Right: M-flame, $\phi=1.04, u_{b}=1.97 \mathrm{~m} \mathrm{~s}^{-1}$. 

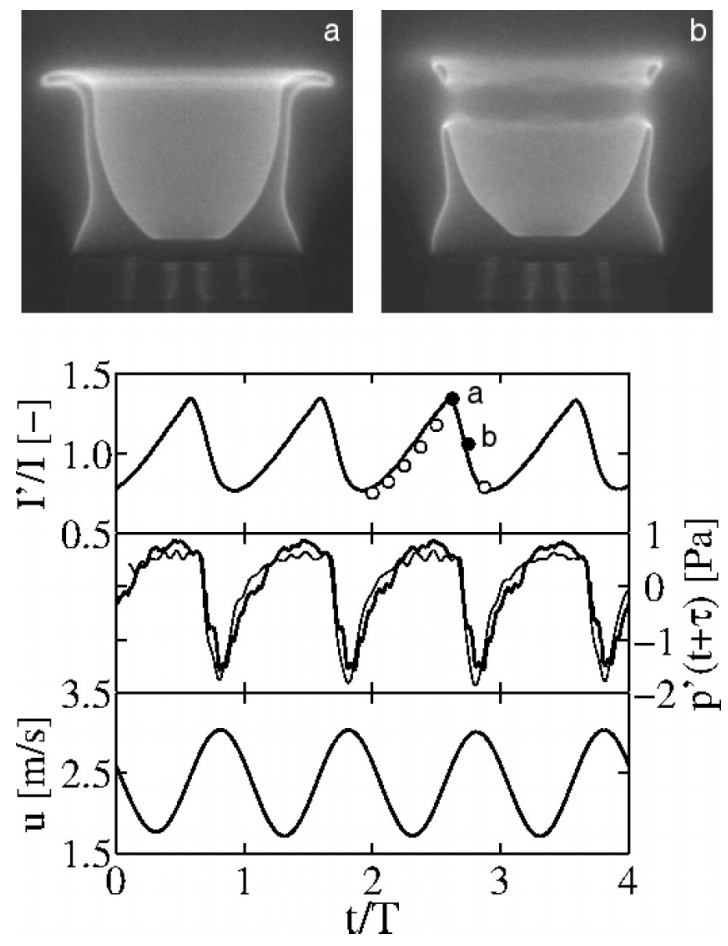

FIG. 3. Top: cyclic flame motion subjected to a flow modulation at $f=150 \mathrm{~Hz}$ with $\phi=1.04, u_{b}=1.97 \mathrm{~m} / \mathrm{s}$, and $u^{\prime} / u_{b}=0.25$. Snapshots are, respectively, taken at $3 T / 2$ (a) and $7 T / 8$ (b), where $T=1 / f$. Bottom: corresponding input velocity signal $u(t)$, acoustic pressure $p^{\prime}(t+\tau)$, and relative heat release rate fluctuations measured by relative flame light $I^{\prime}(t) / \bar{I}$ oscillations as a function of time $t / T$. The delay $\tau=r_{\infty} / c_{0}$ coincides with the sound propagation time. The rate of change $d I^{\prime} / d t(t-\tau)$ (thin line) is also superimposed on the acoustic pressure (thick line).

When forced at a finite amplitude, the M-flame wrinkles as shown in Figure 3. Perturbations are convected along the flame front towards the flame tip. Relative fluctuations of the flame chemiluminescence intensity $I^{\prime} / I$ are plotted as a function of time in Fig. 3 for a harmonic velocity modulation $u^{\prime} / u_{b}=0.25$ at $f=150 \mathrm{~Hz}$, where $u^{\prime}$ denotes the rms-fluctuations of the axial velocity. This signal already contains a large harmonic component and is out of phase with the sinusoidal velocity signal $u$ at the burner outlet. Images (a) and (b) in Fig. 3 correspond to a pinching of the flame front leading to the release of a flame torus. This phenomenon leads to a sudden drop of the flame chemiluminescence intensity $I^{\prime} / I$ in the oscillation cycle. This rapid rate of flame surface destruction corresponds to a pressure peak in the sound pressure $p^{\prime}(t+\tau)$ recorded by the microphone, represented by the thick line in Fig. 3. The time lag $\tau=r_{\infty} / c_{0}$ corresponds to the propagation time of sound waves from the compact source region to the microphone location at the speed of sound $c_{0}=340 \mathrm{~m} / \mathrm{s}$. The pressure predicted from combustion noise theory is represented by the thin line in Fig. 3. This estimate compares well with microphone measurements.

At small forcing amplitudes, effects of the forcing frequency are considered by determining the FTF between the upstream flow velocity and heat release rate disturbances. This response is calculated by only considering the signals at the fundamental frequency associated with the forcing frequency. Heat release rate disturbances were determined by recording the flame chemiluminescence intensity signal $I(t)$ collected by the photomultiplier (PM) equipped with a $\mathrm{CH}^{*}$ interferometric filter. Flame surface, flame light intensity, and heat release rate fluctuations were shown to be proportional, ${ }^{62}$ and one may write

$$
\frac{\widetilde{\tilde{Q}}}{\overline{\dot{Q}}}=\frac{\tilde{I}}{\bar{I}}=G \exp (i \varphi) \frac{\tilde{u}}{u_{b}},
$$

where $\tilde{a}$ denotes the Fourier components of the signal $a(t)$ examined at the forcing frequency and $\bar{a}$ stands for the mean value of the signal. This frequency response was obtained by modifying the 


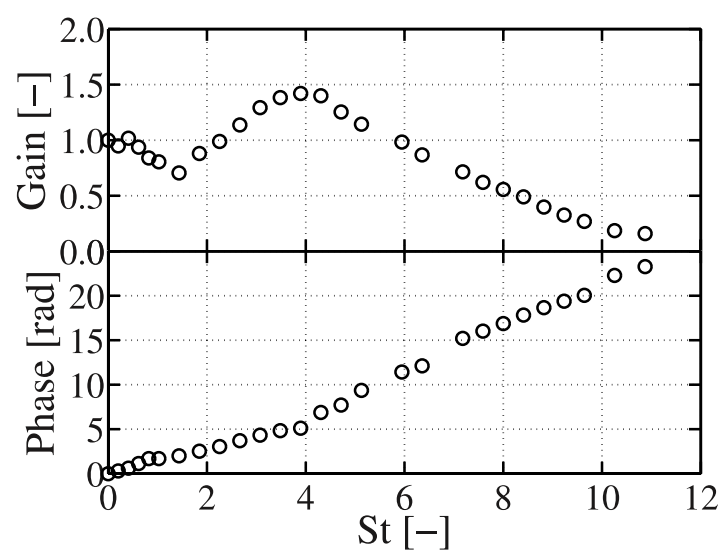

FIG. 4. Gain (top) and phase lag (bottom) of the FTF as a function of the Strouhal number $S t=f\left(r_{1}-r_{0}\right) / S_{d}$ for an M-flame with $\phi=1.04, u_{b}=1.97 \mathrm{~m} / \mathrm{s}, u^{\prime} / u_{b}=0.08$, and $S_{d}=0.39 \mathrm{~m} / \mathrm{s}$.

harmonic forcing frequency for a fixed velocity perturbation level $u^{\prime} / u_{b}=0.08$ at the burner outlet controlled by LDV.

Results for the gain $G$ and phase lag $\varphi$ of the FTF are presented in Fig. 4 in terms of a Strouhal number $S t=f\left(r_{1}-r_{0}\right) / S_{d}{ }^{62}$ where $S t$ denotes the angular forcing frequency and $S_{d}$ the laminar burning velocity of the combustible mixture. The FTF has a gain of unity at very low frequencies. It then takes on values lower than unity and reaches a minimum at about $S t \simeq 1.5$. The gain then increases and takes on values larger than unity for $S t \geq 2$ with a peak of about $G=1.5$ at $S t=4$, indicating the amplification of the flow perturbations by the flame within this frequency range. Higher forcing frequencies are progressively filtered by the flame with a decrease in gain with increasing forcing frequencies. The gain is lower than unity for $S t \geq 6$, and the FTF cutoff frequency, where the gain falls below 0.5 , is equal to $S t \simeq 8$.

The FTF phase lag between velocity and heat release rate disturbances starts from zero and steadily increases with the forcing frequency. One notes an inflection point when the gain reaches the small local minimum near $S t \simeq 1.2$. The phase derivative is almost constant and equal to $v_{\varphi}=1.2 \mathrm{rad} / \mathrm{St}$ when $S t \leq 4$. It then increases and takes on a slightly larger value $v_{\varphi}=2.5 \mathrm{rad} S t^{-1}$ for $S t \geq 4$. This steadily increasing phase lag is characteristic of perturbations convected by the mean flow with a velocity proportional to the bulk injection velocity $u_{b}=1.97 \mathrm{~m} / \mathrm{s}$, see Refs. 6 and 28 .

These responses in terms of FTF and noise radiated by the perturbed flame serve as reference experiments to validate the numerical solver developed below.

\section{NUMERICAL APPROACH}

The numerical approach chosen in this study is based on a linear computation. This technique allows for the study of the perturbations' dynamics without employing a reduced-order model. Strong linear couplings between the reaction layer, hydrodynamics, and acoustics are also preserved. It is assumed that these interactions can account for most physical phenomena involved in thermoacoustic interactions. The numerical code was adapted from a compressible, inert gaseous flow solver developed in Ref. 46. The conservation equations are first presented, followed by the combustion model. Different approximations are introduced in this version of the solver for the description of species transport and combustion. They are listed below. Only the minimum material, needed to capture the response of laminar flames to small flow disturbances, was included in the numerical approach. These assumptions are assumed to retain the most important features of the laminar flame dynamics and are intended as a first step towards more complex models. This approach should highlight the principal physical mechanisms involved in acoustic amplification rather than to lead a close quantitative comparison. 


\section{A. Governing equations}

A viscous, axisymmetric, compressible, reactive laminar flow is considered. Throughout this study, the Lewis number of all species is taken as unity. To simplify the problem, heat capacities are also kept equal and constant for all species. Species diffusion fluxes are modeled by Fick's law. The governing equations for density $\rho$, the velocity field $\mathbf{u}$, total energy $E$, and the $k$ th species mass fraction $Y_{k}$ are given as ${ }^{63}$

$$
\begin{aligned}
\frac{\partial \rho}{\partial t}+\nabla \cdot(\rho \mathbf{u}) & =0, \\
\frac{\partial \rho \mathbf{u}}{\partial t}+\nabla \cdot(\rho \mathbf{u} \otimes \mathbf{u}) & =-\nabla p+\nabla \cdot \underline{\tau}, \\
\frac{\partial \rho E}{\partial t}+\nabla \cdot(\rho \mathbf{u} E) & =\dot{\omega}_{T}-\nabla \cdot \mathbf{q}+\nabla \cdot(\underline{\boldsymbol{\tau}} \cdot \mathbf{u}), \\
\frac{\partial \rho Y_{k}}{\partial t}+\nabla \cdot\left(\rho \mathbf{u} Y_{k}\right) & =-\dot{\omega}_{k}+\nabla \cdot\left(\rho D \nabla Y_{k}\right),
\end{aligned}
$$

where

$$
\dot{\omega}_{T}=\Sigma_{k} \dot{\omega}_{k} \Delta h_{f, k}^{0}
$$

and $p$ is the pressure, $D$ denotes the diffusion coefficient common to all species, $\underline{\tau}$ stands for the stress tensor, $\Delta h_{f, k}^{0}$ is the formation enthalpy of species $k$ at the reference temperature $T_{0}=300 \mathrm{~K}$, and $\dot{\omega}_{k}$ represents the production rate of species $k$. In our case, only the fuel mass fraction is explicitly computed and $k=1$. In what follows, reaction rate and fuel mass fraction will be, respectively, designated with $\dot{\omega}_{f}$ and $Y_{f}$. A Newtonian fluid with viscosity $\mu$, given by Sutherland's law, is considered to link the stress tensor to the velocity flow field. Details on the implementation of these equations for an axisymmetric configuration can be found in Refs. 46 and 64.

\section{B. Chemistry}

A one-step, one-way chemistry was used to model the premixed flame. The flame is supposed to be stoichiometric, even though experiments were conducted at a slightly rich condition with a mixture ratio of $\phi=1.04$. The fuel mass reaction rate is given by an Arrhenius model of the form

$$
\dot{\omega}_{f}=A \rho Y_{f} \exp \left(-\frac{T_{a}}{T}\right),
$$

where $A$ is the Arrhenius prefactor and $T_{a}$ is the activation temperature. These parameters were set to stabilize the flame on the burner at a prescribed displacement speed reproducing the same flame topology as shown in experiments, while keeping the flame thickness within a reasonable range. These conditions were obtained for an Arrhenius prefactor set to $A=6.04 \times 10^{5} \mathrm{St}^{-1}$ and for

$$
\frac{T_{b}}{T_{u}}=6.47 \text { and } \frac{T_{a}}{T_{u}}=40,
$$

where $T_{u}$ denotes the temperature in the injection unit, fixed at $300 \mathrm{~K}$, and $T_{b}$ stands for the temperature of burnt gases. The associated Zeldovitch parameters are accordingly

$$
\alpha=\frac{T_{b}-T_{u}}{T_{b}}=0.845 \text { and } \beta=\alpha \frac{T_{a}}{T_{b}}=5.23 .
$$

These parameters correspond to a flame of thickness $\delta \simeq 0.05 \mathrm{~mm}$ stabilized on a burner of $0.7 \mathrm{~mm}$ in diameter. This flame is also faster $S_{d} \simeq 3.63 \mathrm{~m} / \mathrm{s}$ than the laminar burning velocity $S_{d}=0.39 \mathrm{~m} / \mathrm{s}$ of the methane/air flame considered in the experiments. The computed flame thus admits a bulk injection velocity of $u_{b}=20.79 \mathrm{~m} / \mathrm{s}$, which is rather high for air-combustion hydrocarbon flames, but reasonable for oxy-flames. Stoichiometric methane-oxygen flames have a laminar burning velocity of $3.3 \mathrm{~m} / \mathrm{s}$ close to the value used in the simulations. In what follows, all quantities are expressed with respect to the tube radius $r_{\text {tube }}=0.7 \mathrm{~mm}$, the tube bulk injection velocity $u_{b}$, and the unburned gas temperature $T_{u}=300 \mathrm{~K}$. 
These choices were guided by a compromise between simulations of realistic, laminar flame configurations, and optimization of the computational effort. It allowed for a validation of the methodology, before considering more challenging configurations and chemistries in future studies. It is however worth mentioning that within the range of frequencies considered in this study, the flame remains compact with respect to acoustics. Indeed, the characteristic acoustic wavelength considered here is of order $L / M a$, where $L$ is the flame characteristic length. In our case, $L$ is typically of the order of the tube radius. Furthermore, the Mach number is kept low, with a local value of $M a \leq 0.1$, which enforces a length scale separation that should maintain the validity of our results for more complex cases.

\section{Geometry}

The numerical domain consists of an annular pipe expelling gases into a cylindrical region shown in Figs. 5 and 6. The combustible mixture is injected through the annular pipe of internal radius $r_{0}=0.27$ and external radius $r_{1}=1$ and burns at the exit. This computational domain was split into two regions, the first one containing the annular pipe and the second one containing the exit region. The burnt gases expand into the atmosphere via absorbing boundary conditions. The fresh reactants are injected through the annular tube which has been taken sufficiently long to contain one full period of an acoustic wave at the characteristic flame frequency $S_{d} /\left(r_{1}-r_{0}\right)$, where $S_{d}$ is the laminar burning velocity. In what follows, flame front (a) will be designated as the "internal flame front," while flame front (b) will be referred to as the "external flame front."

Using the bulk injection velocity $u_{b}$ and the outer radius $r_{1}$ as reference scales, the corresponding Reynolds number has been set to $R e=950$, and the bulk Mach number in the tube at $T_{u}$ to $M a=0.065$ for a maximum local injection mach number of $M a_{\max }=0.1$. The maximum base-flow velocity at the injection plane was measured as $u_{\max }=1.54$. Compared to the reference experiment, this sets the Reynolds number of our simulation lower by a factor of 2 and the injection Mach number one order of magnitude higher. The choice of flow parameters and geometric dimensions, however, has been driven by computational limitations. In fact, the Courant-Friedrichs-Lewy (CFL) condition locally imposes ${ }^{65}$

$$
d t \sim \frac{d x}{U_{0, \text { loc }}\left(1+\frac{1}{M a_{l o c}}\right)}
$$

for compressible computations, which imposes a rather stringent constraint on the Mach number.

In the following, we use the same Strouhal number to allow a compare between numerical results and experimental data. We thus define

$$
S t=\frac{f\left(r_{1}-r_{0}\right)}{S_{d}} .
$$

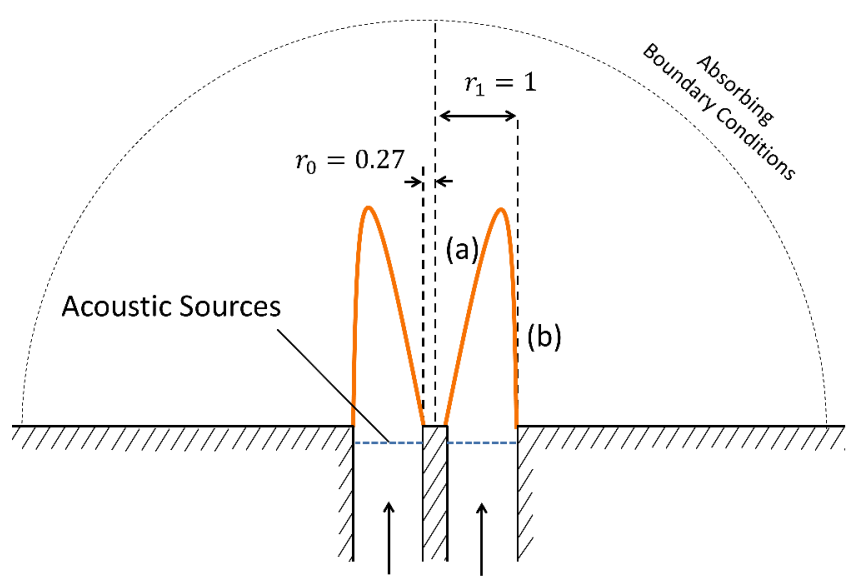

Premixed fuel

FIG. 5. Sketch of the numerical configuration. 


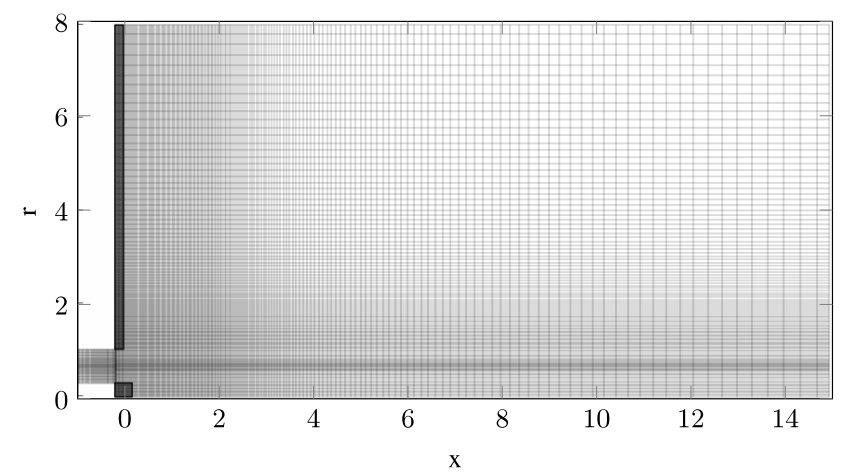

FIG. 6. Typical mesh used in simulations, coarsened four times in each direction. The shaded regions are treated as non-slipping walls.

\section{Numerical details}

Spatial derivatives have been computed to third-order accuracy using a 11-point stencil, optimized in the Fourier domain. ${ }^{66}$ Time integration has been performed using a 10-step, strongly stability-preserving, fourth-order Runge-Kutta method. ${ }^{67,68}$ The flame has been fully resolved. For nonlinear simulations, an operator-splitting method has been used to solve for the chemistry. At each intermediate Runge-Kutta step, the following partial system is employed:

$$
\begin{aligned}
& \frac{\rho E(t+d t)-\rho E(t)}{d t}=\Delta h_{f}^{0} \dot{\omega}_{f}(t+d t), \\
& \frac{\rho Y_{f}(t+d t)-\rho Y_{f}(t)}{d t}=-\dot{\omega}_{f}(t+d t) .
\end{aligned}
$$

This system is solved for $\left(\rho E(t+d t), \rho Y_{f}(t+d t)\right)$ using a Newton-Raphson technique, based on the time step $d t$ of the global Runge-Kutta step. The resulting value of $\dot{\omega}_{f}(t+d t)$ is then used in Eq. (5). This method was observed to yield consistent, stable results for the range of time steps typically encountered in our simulations. In the worst case, this scheme locally decreases the temporal accuracy of the simulation to first order for the chemistry terms. This apparent loss in precision, however, has no impact on subsequent simulations, since the nonlinear computations were only used to establish a steady base flow. The discretized system contained a total of 2,785,280 degrees of freedom, and simulations were conducted at a CFL number of 1.18. An implementation on parallel architectures has been handled via the PETSc library. ${ }^{69,70}$

\section{E. Base flow}

In order to perform a linearized simulation, a steady-state solution is required. This base flow represents the solution of the system,

$$
\begin{aligned}
\nabla \cdot\left(\rho_{\mathbf{0}} \mathbf{u}_{\mathbf{0}}\right) & =0, \\
\nabla \cdot\left(\rho_{0} \mathbf{u}_{\mathbf{0}} \otimes \mathbf{u}_{\mathbf{0}}\right) & =-\nabla p_{0}+\nabla \cdot \underline{\boldsymbol{\tau}_{\mathbf{0}}}, \\
\nabla \cdot\left(\rho_{0} \mathbf{u}_{\mathbf{0}} E_{0}\right) & =\dot{\omega}_{f, 0} \Delta h_{f}-\nabla \cdot \mathbf{q}_{\mathbf{0}}+\nabla \cdot\left(\underline{\boldsymbol{\tau}_{\mathbf{0}}} \cdot \mathbf{u}_{\mathbf{0}}\right), \\
\nabla \cdot\left(\rho \mathbf{u}_{\mathbf{0}} Y_{f, 0}\right) & =-\dot{\omega}_{f, 0}+\nabla \cdot\left(\rho D \nabla Y_{f, 0}\right) .
\end{aligned}
$$

In all cases explored in this study, the nonlinear simulation showed that the flow is naturally convectively unstable. Indeed, the flame produces a hot shear layer associated with a Kelvin-Helmholtz instability. This is not surprising as hot jets at these working conditions are typically either convectively or absolutely unstable. ${ }^{71-74}$ This instability mostly influences the hot products downstream of the flame; it was further assumed to have a negligible impact in this study. In order to suppress this unsteady dynamical component, a selective frequency damping (SFD) technique ${ }^{75}$ has been applied. To this end, two linearly coupled equations are simultaneously advanced in time: the first equation 


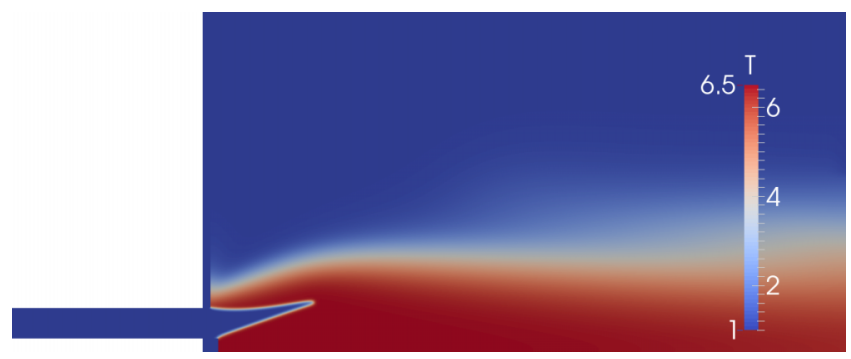

FIG. 7. Base flow used for the linearization procedure, visualized by temperature ratio $T / T_{u}$ with $T_{u}=300 \mathrm{~K}$.

associated with the field variables and the second equation describing an observer state. The observer is damped such that the dynamics of the field variable no longer retains the unstable, unsteady state. It is readily verified that upon convergence of the procedure, a steady state of the nonlinear reactive compressible Navier-Stokes equations is obtained. This solution has been taken as the base flow and is presented in Fig. 7, visualized by temperature. In this figure, the shear layer is confined to the mixing layer (in grey) away from the flame.

\section{LINEAR RESPONSE ANALYSIS}

\section{A. Linearization}

We proceed by carrying out a full, exact linearization about our base flow, using the direct numerical linearization technique developed in Ref. 76 which allows an efficient implementation and evaluation of the linear operator (about a given base flow) underlying a nonlinear numerical simulation. For the case of a reacting compressible flow, following a reordering of matrix components, this procedure provides numerical access to the linearized operator $A_{\text {full }}$, split into respective physical mechanisms. For example, we obtain

$$
A_{\text {full }}=A_{\text {flow }}+A_{\text {chem }}
$$

where $A_{\text {flow }}$ is the operator associated with the compressible non-reactive flow dynamics, while $A_{\text {chem }}$ is the operator associated with the linearization of $\dot{\omega}_{T}$ and $\dot{\omega}_{f}$ in Eqs. (4) and (5). It is worth noting that in a linearized simulation, the state vector is defined up to a multiplicative constant. This justifies the use of dimensionless numbers to express results.

\section{B. Impulse response analysis of the system: The transfer function}

In order to evaluate the effects of acoustic waves on the flame, a planar acoustic forcing is employed. The acoustic source distribution is presented in Fig. 5. The acoustic forcing is introduced as a set of source terms, uniform in the $r$-direction, with a Gaussian-shaped amplitude, centered about $x=-0.1$ upstream of the injection plane and with a characteristic width of 0.02 , i.e.,

$$
f(r, x)=\left(\begin{array}{c}
\rho_{f}(x) \\
\rho_{f}(x) u_{0}(r, x) \\
\rho_{f}(x) v_{0}(r, x) \\
\rho_{f}(x)\left(c_{0}^{2} /(\gamma-1)+0.5\left(u_{0}^{2}+v_{0}^{2}\right)\right)(r, x) \\
\rho_{f}(x) Y_{0}(r, x)
\end{array}\right),
$$

with

$$
\begin{aligned}
\rho_{f}(x) & =\frac{\rho_{l o c}(x)}{\int_{-\infty}^{+\infty} \rho_{l o c}\left(x^{\prime}\right) d x^{\prime}}, \\
\rho_{l o c}(x) & =\exp \left(-(x+0.1)^{2} / 0.02^{2}\right) .
\end{aligned}
$$


The above expressions define a set of density sources acting at constant entropy; such a set has been found to generate nearly pure, acoustic waves. The response of the flame to this forcing is determined by computing a numerical impulse response. In experiments, the linear flame response is typically inferred frequency-by-frequency using an harmonic forcing as in Fig. 4. In numerical simulations, a low-amplitude broadband signal, such as a random binary signal, can be applied to identify the system impulse response. ${ }^{25}$ In our case, initial conditions have been applied using Eq. (19); in particular, a volume forcing is applied at $t=0$ and thereafter set to zero, and the propagation of the resulting acoustic impulse is computed. Applying a Fourier transform to this impulse response yields the transfer function to our particular forcing defined by Eq. (19).

To allow comparison with experiments, this transfer function has been further modified according to

$$
(\rho u)_{b}^{\prime}=\frac{1}{\pi\left(r_{1}^{2}-r_{0}^{2}\right)} \int_{r_{0}, x=-0.05}^{r_{1}} 2 \pi r(\rho u)_{x}^{\prime}(x, r) d r
$$

as an input. In experiments with $M a \ll 1$, we have $\tilde{u} / u_{0} \gg \tilde{\rho} / \rho_{0}$ and $(\widetilde{\rho u}) /(\rho u)_{0} \simeq \tilde{u} / u_{0}$. In our case, with $M a=0.1$, the density term has been retained; it introduces a correction of typically two percent to the transfer function. In addition, the transfer function, for a field variable $g$, has been formulated in the form

$$
T F_{g}=\frac{\tilde{g} / g_{0}}{(\widetilde{\rho u})_{b}^{\prime} /(\rho u)_{b, 0}},
$$

where $\tilde{g}$ denotes the Fourier transform of the impulse response of $g$.

In Fig. 4, the FTF consists of the computation of the transfer function associated with the relative $\mathrm{CH}^{*}$ light intensity using the relative bulk velocity perturbation as an input. A numerical simulation allowing a direct access to the heat release rate, the relative variations of this quantity was used as an output here. For this reason, the variable $g$ in the expression above has been taken as the volumetric integral of the heat release rate $\dot{q}$, i.e.,

$$
g=\dot{\mathrm{q}}=\int_{\Omega} \dot{\omega}_{T} d \Omega,
$$

where $\Omega$ stands for the computational domain. The corresponding transfer function $T F_{q}$, presented in Fig. 8, thus quantifies flame sensitivity to acoustics in the limit of infinitesimal perturbations.

Figures 4 and 8 can now be easily compared. Experiments show an asymptotic value of one for the transfer function as $S t \rightarrow 0$, with a local minimum at $S t=1.5$ and a local maximum at $S t=4$; the phase is linearly increasing over the same range of frequencies, at a rate of $2.5 \mathrm{rad} S t^{-1}$. The linear variation of the phase, present in both Figs. 4 and 8, is characteristic of the phase delay $\varphi$ associated with wave propagation of the form

$$
\varphi=k x=\frac{2 \pi S t}{U_{c}} x,
$$
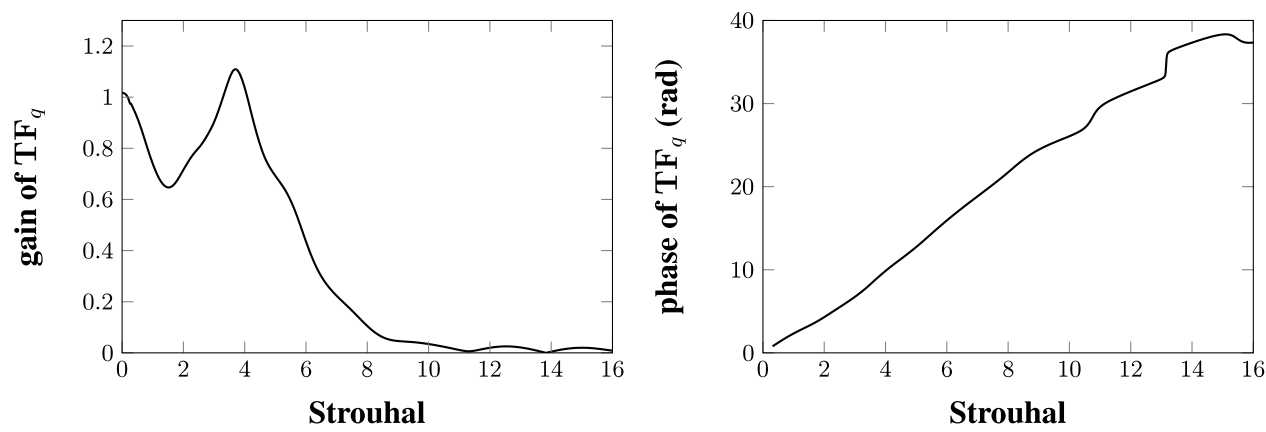

FIG. 8. Amplitude and phase of the computed flame transfer function. 

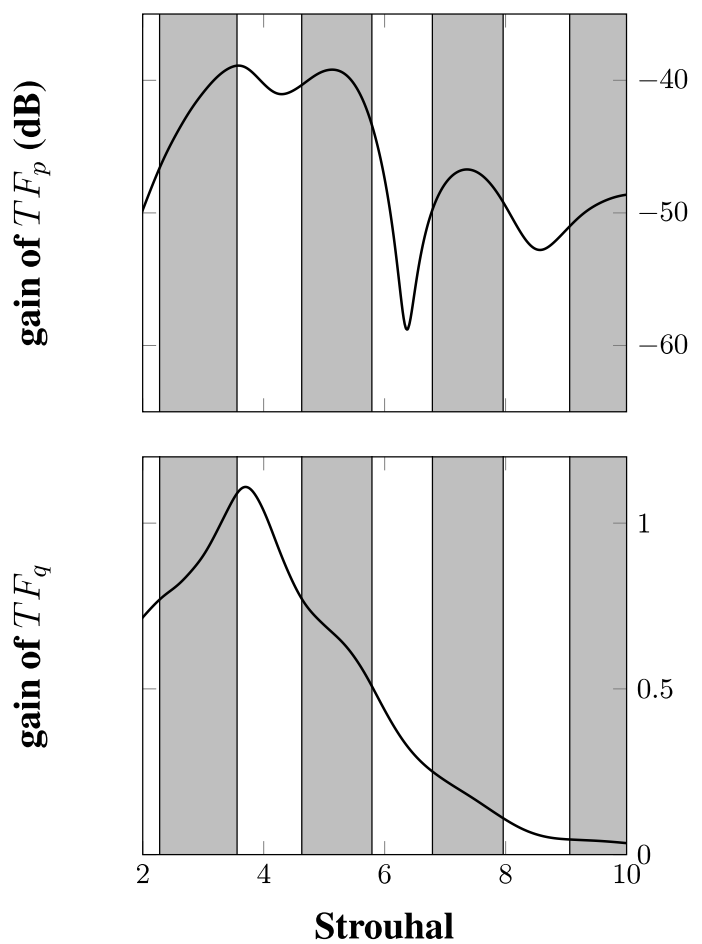

FIG. 9. Top: pressure gain (in $\mathrm{dB}$ ) at $\mathrm{x}=4, \mathrm{r}=1.5$; bottom: gain of the flame transfer function. Grey areas: frequency ranges corresponding to expected constructive interactions between heat release rate and pressure based on the Rayleigh's criterion.

where $U_{c}$ is the characteristic velocity of the wave considered. Here, we have $U_{c} \approx 1$ which corresponds to a hydrodynamic velocity. The predicted phase is also linear in frequency with a coefficient proportional to the distance $x$ from the injection plane. These relations are correctly predicted by the numerical simulation; however, quantitative differences appear in the values of the gain for the local maximum and minimum, as well as in the rate of decrease of the gain past its maximum. This may be attributed to insufficient accuracy of our chemistry model, to the length-scale separation between the simulated and the real flame, or to the finite amplitude of the perturbations used in experiments. As this study focuses on the physical mechanisms associated with flame/acoustic interactions, the above results seem adequate to validate our approach.

According to Rayleigh's criterion, if the unsteady rate of heat released is positively correlated to the pressure of the oscillation, thermoacoustic amplification will occur. We have

$$
\lambda \propto \int_{0}^{2 \pi / \omega} p_{\text {in }} \dot{q} d t
$$

with $\lambda$ as the pressure amplification and $p_{i n}$ as the forcing pressure. As presented in Fig. 9, an evaluation of the integral unsteady heat release phase and gain also allows the prediction of frequency ranges where amplification can be observed. However, this provides little information on which spatial regions are subject to large acoustic amplification due to chemistry. In addition, we also wish to identify (and visualize) the acoustic sources induced by unsteady chemistry.

Generating such a map in space for a given frequency requires us to compare pressure levels at different points, and in doing so, compensation for propagation effects is necessary. In the far-field region, such comparisons are typically achieved using asymptotic expressions; in the near field, however, a different approach has to be used. Additionally, our strategy aims at isolating chemical unsteady heat release effects from linear computations, which ultimately allows for an isolation of the acoustic field induced by unsteady chemistry. We therefore perform two sets of computations: a first simulation with (linear) chemistry and a second one without reactive terms. In linear numerical studies, the base flow is typically assumed constant throughout the simulation, and ignoring flame 


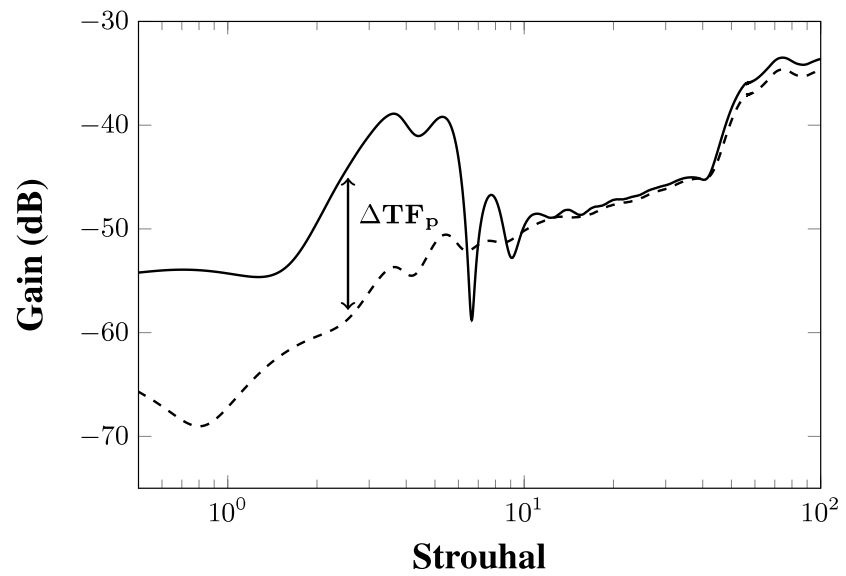

FIG. 10. Comparison of the acoustic gain of $-\operatorname{Tf}_{p, f u l l}$ with chemistry and $-\operatorname{Tf}_{p \text {,partial }}$ without linearized chemistry at $\mathrm{x}=4$, $\mathrm{r}=1$.

effects on the linear acoustics is equivalent to keeping a reacting baseflow (with strong temperature gradients) while switching off linear chemistry terms.

Following the previous formalism, the matrix

$$
A_{\text {full }}=A_{\text {flow }}
$$

is then used instead of Eq. (18), while keeping all other simulation parameters identical. Applying our system analysis technique to the full system with (18) then yields the full transfer function $T F_{p, f u l}$, while using (27) leads to the transfer function without linear chemistry $T F_{p, p a r t i a l}$. The ratio between these two transfer functions, i.e.,

$$
\Delta T F_{p}=\frac{T F_{p, \text { full }}}{T F_{p, p a r t i a l}}
$$

then characterizes the amplification of acoustic waves by the flame, without being influenced by propagation effects or by interactions with the hydrodynamics.

Figure 10 presents, at the point $\mathbf{x}=(4,1)$, the transfer functions $T F_{p, \text { full }}$ and $T F_{p \text {,partial, as well }}$ as a graphic interpretation of $\Delta T F_{p}$. Two frequency ranges can be distinguished: for $S t<6$, strong acoustic amplifications due to unsteady chemistry occur, while for $S t>6$, both transfer function curves coincide. This observation is in agreement with the flame transfer function shown in Fig. 8. At high forcing frequencies, the flame saturates and no amplification is observed; the same behavior is observed in Fig. 4.

\section{Acoustic amplification map}

Instead of considering the variation of acoustic amplification with frequency at a given location, our procedure allows for the comparison of the transfer function $\Delta T F_{p}$ for varying points in space at a prescribed frequency. This results in a spatial map of acoustic amplification, such as presented in Fig. 11, for $S t=3.54$ and $S t=10.58$.

At both frequencies, the spatial structures related to acoustic amplification are comparable. However, the amplitudes in both cases are drastically different. Confirming previous observations, at $S t=3.54$, the gain reaches a maximum of $25 \mathrm{~dB}$, while at $S t=10.6$, a maximum of $1.7 \mathrm{~dB}$ is obtained. Moreover, these local maxima of amplification are located at the flame tip, which is in agreement with experimental light intensity measurements in Figs. 3(a) and 3(b). Indeed, the local nonlinear heat release rate at the flame front can be expressed as

$$
d \dot{q}=\rho_{1} S_{f} \Delta h \mathrm{dA},
$$

with $\rho_{1}$ as the upstream density, $S_{f}$ as the normal flame front speed, defined with respect to the flow, $\Delta h$ as the mass heat of reaction, and $d A$ as an infinitesimal flame surface element. According to 


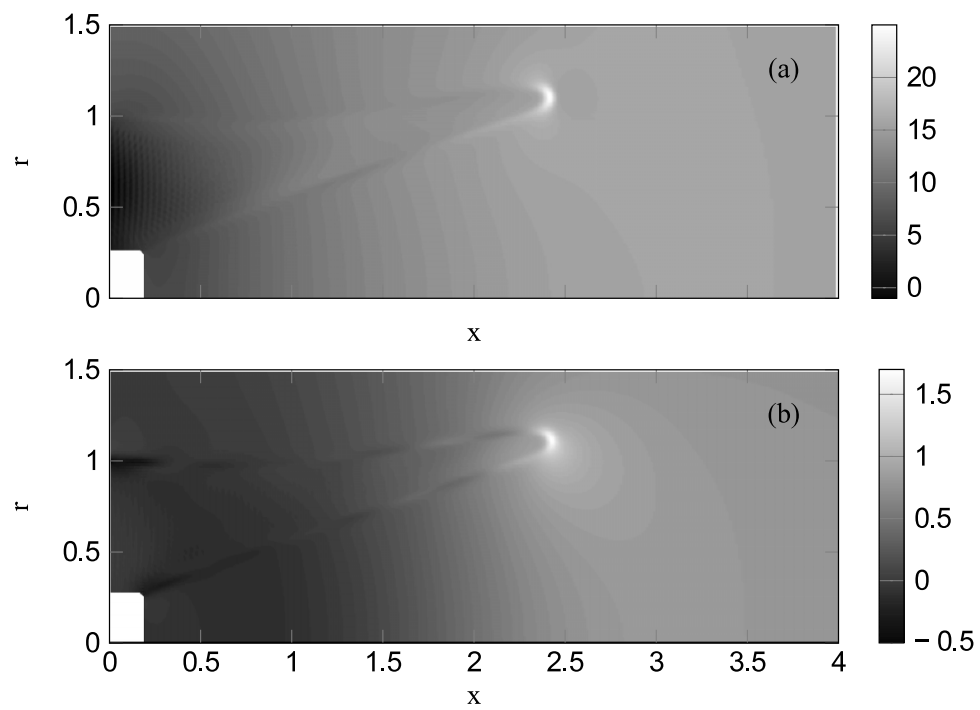

FIG. 11. Mapping of the pressure amplification attributed to unsteady chemistry $\Delta T F_{p}$. (a) $S_{t}=3.54$; (b) $S_{t}=10.58$. Gains are expressed in $\mathrm{dB}$.

Markstein's asymptotic analysis, the flame speed mostly depends on stretch, which in turn suggests that the heat release rate will be particularly affected by disturbances at regions of maximum curvature. Dilatation effects will thus be important at the flame tip and result in high acoustic amplitudes. The above figures also underline the necessity of taking local inhomogeneities of heat release into account when estimating interactions between the flame and acoustics. In particular, an approach solely based on flame-surface computations may underestimate unsteady heat-release amplitudes by a significant amount. ${ }^{77,78}$

Our operator decomposition method has the capability of delineating various physical mechanisms, which is certainly not restricted to only the estimation of pressure amplification. For instance, for flame instabilities it can isolate hydrodynamic effects from chemistry influences and help guide an analysis of the most prevailing mechanisms and physical processes. In our present study, this strategy has identified vorticity as a crucial component in the linear flame-front dynamics.

\section{ACOUSTICALLY INDUCED VORTICITY AT THE FLAME FRONT}

\section{A. Convective modes upstream of the flame}

When a flame is acoustically forced, a coherent velocity mode appears in the region between the internal and external flame fronts, as shown in Fig. 12. The phenomenon appears either with (left subfigure) or without (right subfigure) unsteady chemistry. In what follows below, this mode is referred to as the flame-induced oscillatory mode (FIOM). The rise of this mode has been reported in experiments by Baillot et al. ${ }^{60}$ for the case of a conical flame. Birbaud et al. ${ }^{34}$ studied the change in apparent phase speed of the upstream velocity with frequency. Two characteristic behaviors have been observed with (i) a slow convective mode and (ii) a quick acoustic forcing. The transition between both modes in space could be very abrupt and even associated with locally negative phase velocities. In this present study, the same approach is employed. The variation of the upstream values of the velocity has been interpolated from impulse response data on a line presented in Fig. 13. The obtained velocity was then projected onto this line and normalized according to

$$
T F_{F I O M}(\tilde{s}, S t)=\frac{\tilde{u}_{t}(\tilde{s}, S t)}{\tilde{u}_{t}(\tilde{s}=0, S t)},
$$

where $\tilde{s}$ is the local arclength of the line, taken from upstream to downstream. The resulting transfer function $T F_{F I O M}$ is then of the form $\left|T F_{F I O M}\right| \exp (i \varphi)$. Assuming that $\tilde{u}$ is linked to a propagating 

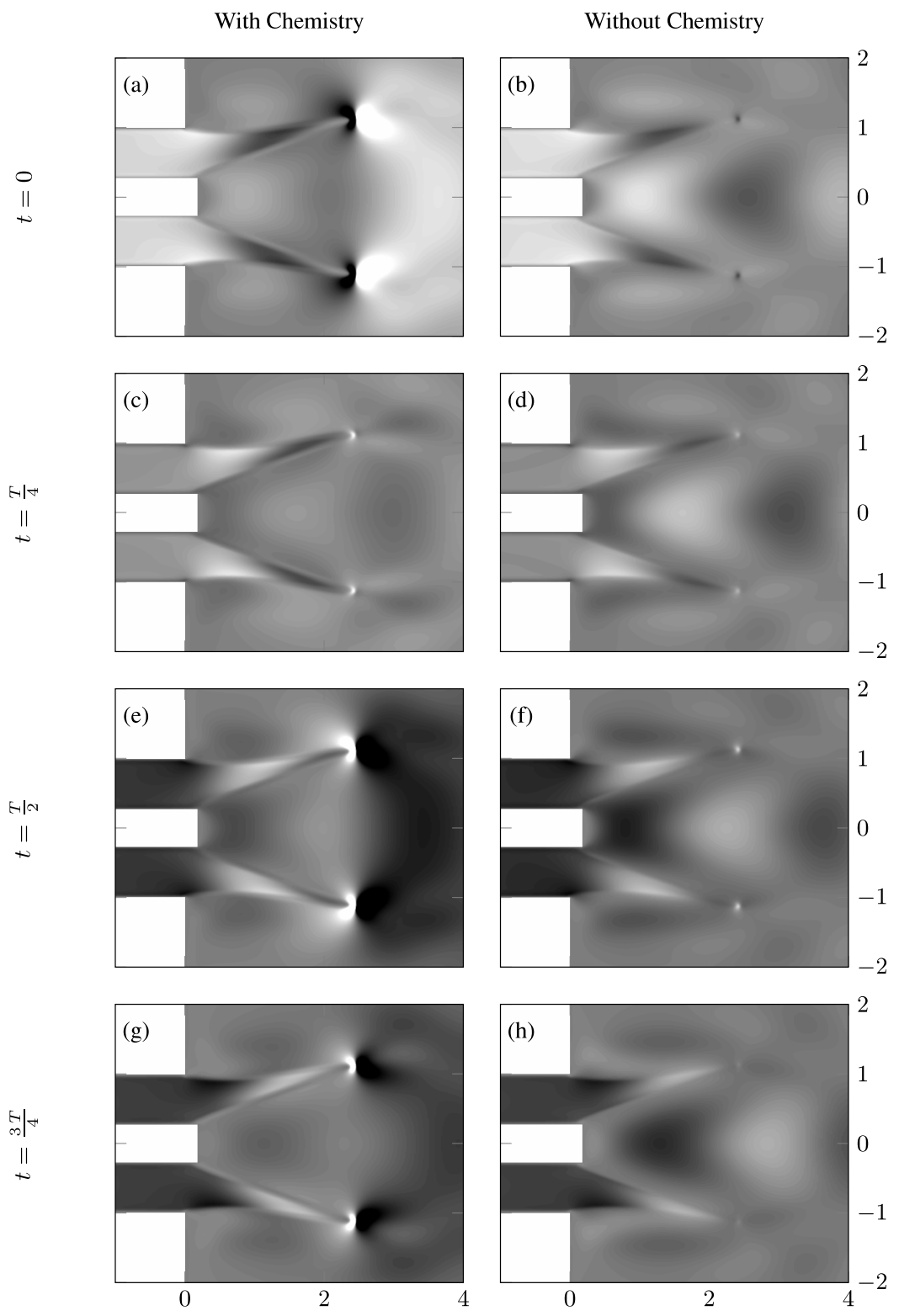

FIG. 12. Longitudinal velocity for a linear forcing at $\mathrm{St}=2.64$, in established regime, with (left) and without (right) linearized chemistry. The colormap is identical for all figures.

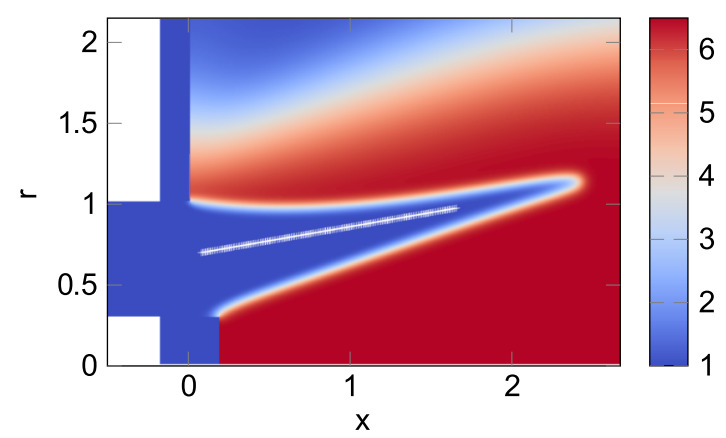

FIG. 13. +symbols denote positions of the extracted points (in white) for the characterization of the FIOM. Background colors are based on the temperature. 


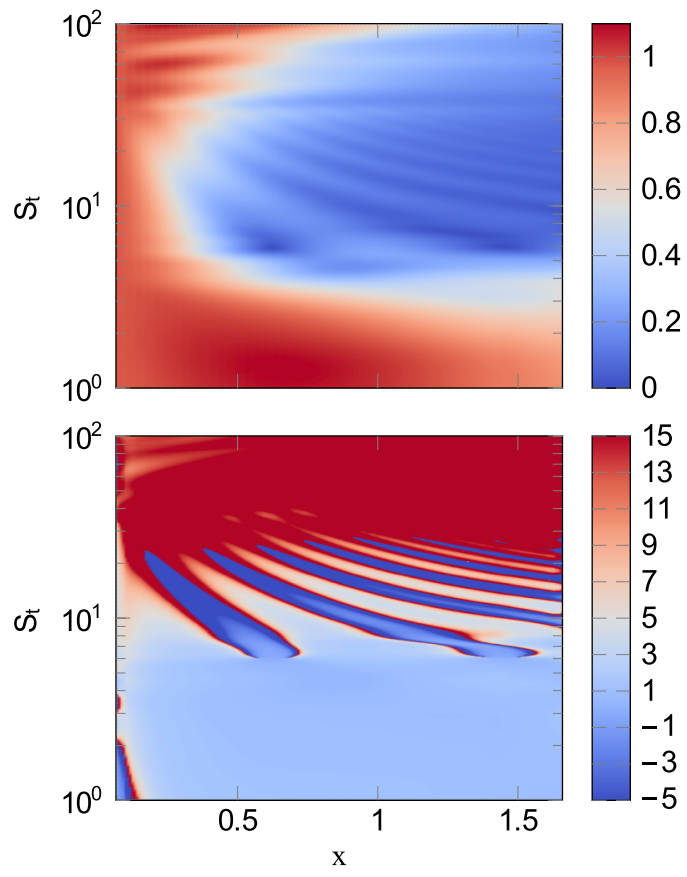

FIG. 14. Norm (top) and phase speed (bottom) of the $\mathrm{TF}_{F I O M}$ computed from the impulse response.

wave allows us to define a pseudo-phase speed as

$$
v_{\varphi}=\frac{\omega}{\partial \varphi / \partial \tilde{s}} .
$$

The results of this decomposition (in norm and phase velocity) are presented in Fig. 14. Data is shown as a function of the axial position $x$ and of the Strouhal number defined as previously.

Typically, acoustic waves are faster than convective waves by a factor of $1 / M a$, equal to ten in our study. A phase speed of order one will thus be labelled as "convective," while a phase speed of order ten will be referred to as "acoustical." Three behaviors can be distinguished. (i) At low Strouhal numbers $(S t<6.3)$, a slow, convective mode dominates, characterized by dark blue regions in the phase-speed plot and by a stable amplitude in space. For instance, at $S t=5$, the phase speed is essentially constant and equal to 1.5 with a slowly varying gain. (ii) For mid-range Strouhal numbers $(6.3<S t<32)$, the amplitude of the velocity decreases along the line, as the convective wave vanishes. A convective and acoustic wave co-exist, which leads to locally negative phase speeds in agreement with previous observations. ${ }^{34}$ Diagonals in the phase velocity map appear. They are related to the interference either between acoustic waves reflected by the flame surface or between acoustic and convective waves. In this regime, the flow becomes gradually dominated by the acoustic forcing as the frequency increases. This tendency is visualized by a shift in background turning from blue to red at $6.3<S t<32$ in Fig. 14. (iii) For very high frequencies ( $S t>32$ ), a detailed study of phase speeds shows that the flow is dominated by unreflected, propagating acoustic waves.

A small region of negative phase speeds appears at very low frequencies. It is believed that this region is associated with the frequency cutoff of the FIOM at low Strouhal numbers.

Given the richness of this dynamics, a direct analysis appears difficult. However, using the analysis technique developed in Sec. IV, we are able to isolate the effects of unsteady chemistry.

\section{B. Identification of vorticity sources}

In this section, we focus on the low-frequency regime. All computations have been performed at $S t=2.64$, and unsteady chemistry has been considered. 

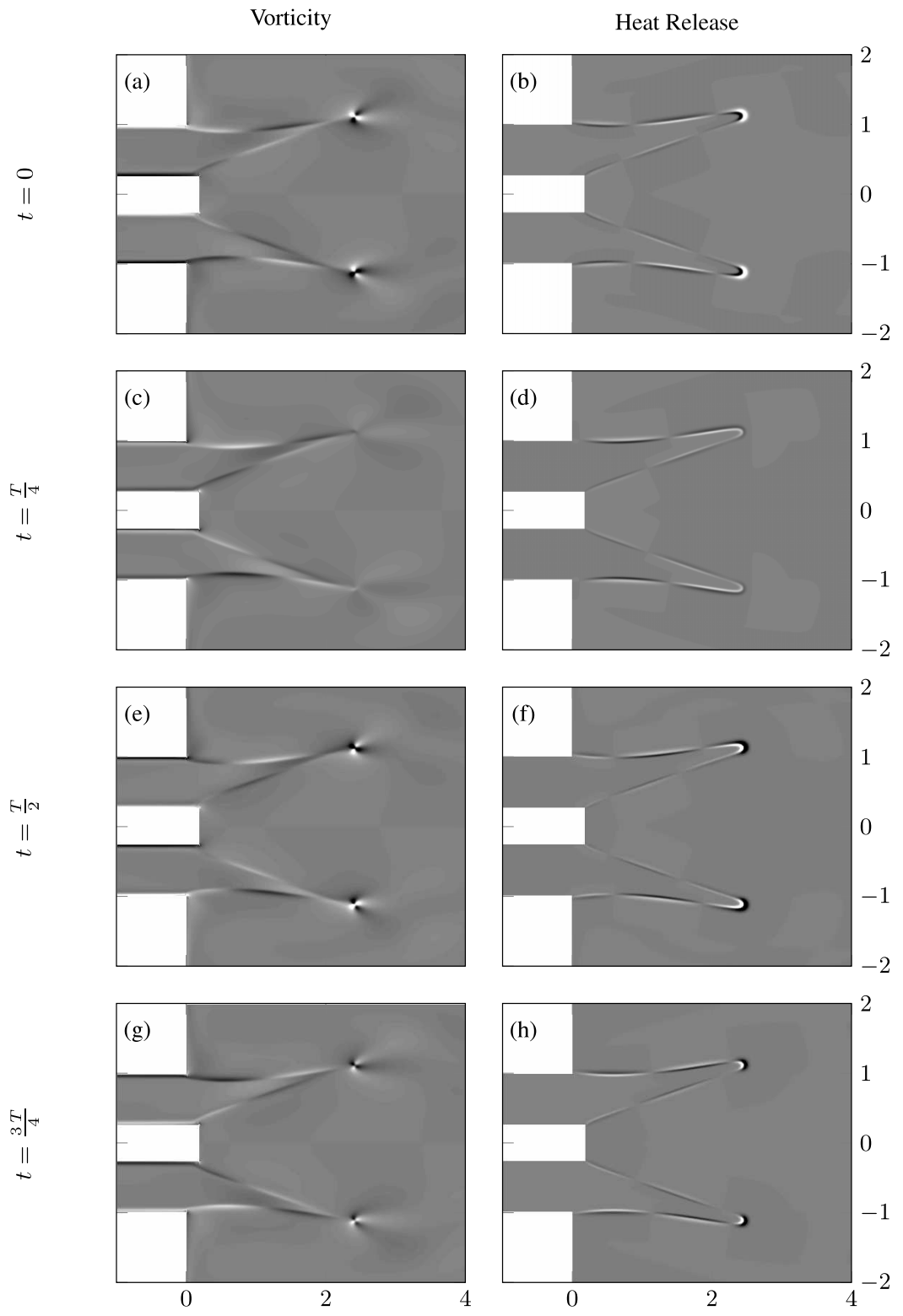

FIG. 15. Vorticity field and unsteady heat release rate for a linear forcing at $\mathrm{St}=2.64$, in established regime. Colormaps are identical, respectively, for all vorticity plots (between -3 and 3 ) and for all heat release rate plots (between -5000 and 5000 ).

In Fig. 15, the presence of a vorticity sheet confined to the flame-front region can be observed. As the flame is periodically pulsed, the internal and external flame fronts are in quasi-phase opposition; this situation is illustrated in Fig. 16.

Through their interaction, the two vorticity sheets induce a dominant longitudinal velocity, confined inside the flame region. The vortices are convected by the flow along the flame fronts. This induces and sets the speed for a convective mode appearing between the internal and external flame fronts. The same mechanism also seems to provide a satisfactory explanation for the FIOM.

In unsteady simulations with linear chemistry, the phase speeds of heat release and of vorticity dynamics are closely correlated, as shown in Fig. 15. This suggests that the above flame-induced vorticity is related to the flame-front dynamics. Moreover, in Fig. 12, a hydrodynamic velocity mode appears, both with and without linearized chemistry, in the region between the internal and external flame fronts, with a notable amplitude in all computations. The mechanism underlying the FIOM 


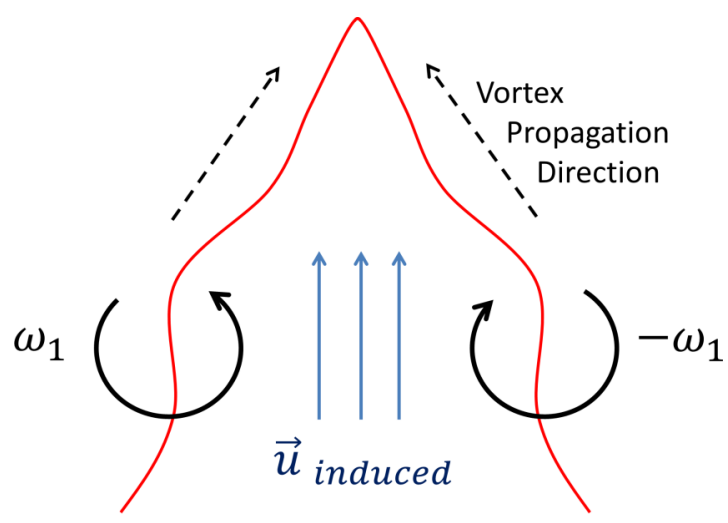

FIG. 16. Effects of flame wrinkling-induced vorticity on the upstream flow.

seems to be of a hydrodynamic nature, since its appearance only weakly depends on the unsteady chemistry associated with the flame. It can further be postulated that the FIOM is linked to the dynamics of the temperature discontinuity present in the base flow. To verify this latter hypothesis, an idealized model is considered. By presuming diffusion effects to be negligible $(\operatorname{Re} \rightarrow \infty)$, the flame becomes infinitely thin - a limit that allows the modeling of the flame using a $G$-equation.

\section{Analytical development of the flame dynamics}

The $G$-equation is based on a level-set method to represent the flame, where the flame front is identified as the zero-level of a globally defined function $G$, with $G>0$ in the region of burned gases and $G<0$ for fresh gases. This zero-level moves at a speed $S_{d}$ normal to the flame front which leads to the equation

$$
\frac{\partial G}{\partial t}+(\mathbf{u} \cdot \nabla) G=S_{d}|\nabla G|
$$

on the flame front. In this formulation, the driving flame-front velocities are taken upstream of the flame.

As we adopt a perturbative approach, the variations of $G$ around a steady position $G_{0}$ are considered next. To this end, all fields $f$ are decomposed according to $f=f_{0}+f^{\prime}$ with $f_{0}$ as the base value of $f$ and $f^{\prime} \ll 1$ as an infinitesimal perturbation. Under the assumption that the flame front does not intersect itself, it is possible to define a local frame of reference $\left(\mathbf{t}_{0}, \mathbf{n}_{0}\right)$, with associated coordinates $(X, Y)$, such that $\mathbf{t}_{0}$ is tangent to the flame front and $\mathbf{n}_{0}$ is normal to it. The normal vector $\mathbf{n}_{0}$ points from fresh to burnt gases, such that $\mathbf{S}_{\mathbf{d}, \mathbf{0}}=-S_{d, 0} \mathbf{n}_{0}$. A sketch of this geometric setup is depicted in Fig. 17. The projection of the speed onto $\left(\mathbf{t}_{0}, \mathbf{n}_{0}\right)$ is denoted by $(u, v)$.

Working in this frame of reference, it can be further deduced that $\partial G_{0} / \partial \mathbf{n}_{0}=1$ for $G_{0} \in \mathscr{C}^{1}$. Thus, at first order, we have

$$
\begin{aligned}
& G \simeq G_{0}-\xi(s, t), \quad \xi \ll 1, \\
& \frac{\partial \xi}{\partial t}+u_{0} \frac{\partial \xi}{\partial s}-v^{\prime}=-S_{d}^{\prime} .
\end{aligned}
$$

In practice, this model corresponds to a geometrically unchanged surface of discontinuity, given implicitly by $G_{0}=0$, for which jumps depend on Eq. (34). This jump also represents the feedback of the flame dynamics on linear perturbations. Equation (34) is the trace of the base-flow discontinuity dynamics. In this model, unsteady chemistry is only accounted for via the forcing term $S_{d}^{\prime}$. Besides, it is well-known ${ }^{79}$ that the flame structure and, in particular, the flame speed only depend on stretch and the equivalence ratio, in the limit of low stretching. This stretch is the consequence of flame curvature and of upstream velocity surface divergence. ${ }^{63}$ In the absence of fuel perturbations, unsteady heat release only has a weak effect on the flame dynamics modeled by Eq. (34). Any mechanism arising from this equation and involving weak relations to $S_{d}^{\prime}$ would also be compatible with the FIOM and 


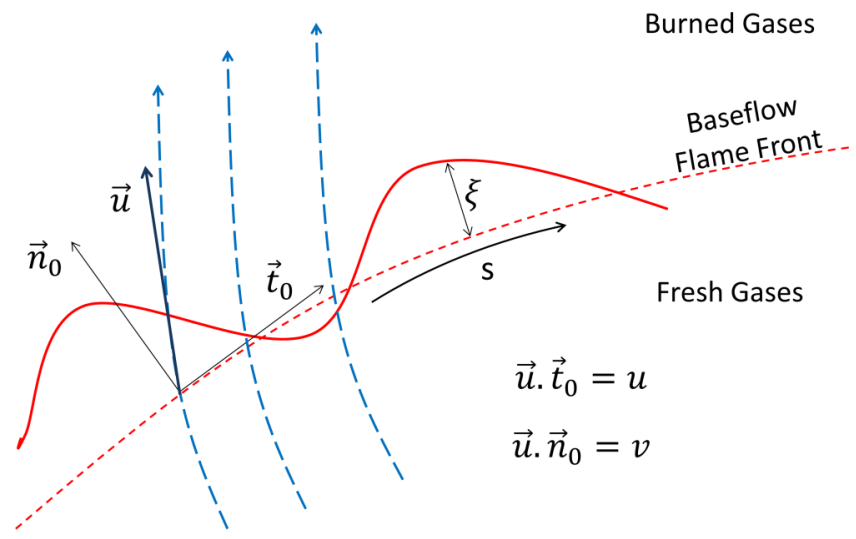

FIG. 17. Conventions for the linearized $G$-equation.

its appearance in all our simulations. In particular, the continuity of the nonlinear tangential velocity

$$
u_{2}-u_{1}=0
$$

leads in our linear framework to the expression

$$
u_{2}^{\prime}-u_{1}^{\prime}=-\frac{\partial \xi}{\partial s}\left(v_{0,2}-v_{0,1}\right) .
$$

This also implies that, at long distance, a wrinkled flame surface acts as a vorticity sheet of local perceived intensity $\partial \xi / \partial s\left(v_{0,2}-v_{0,1}\right)$. This result is rather classical and has been accounted for in Refs. 54 and 80 for the modeling of the Landau-Darrieus instability. Its effect on a planar flame has also been well-studied and reported. However, its influence on a flame composed of two flame fronts and a flame tip is not as well understood.

In order to validate the role of vorticity in the onset of the FIOM, Eqs. (34) and (36) have been implemented in the form of a simplified simulation.

\section{Implementation of the G-equation}

Equation (34) was further developed, based on Ref. 63, to read

$$
\frac{\partial \xi}{\partial t}+u_{0} \frac{\partial \xi}{\partial s}-v^{\prime}=-\kappa_{1} S_{d, 0} C_{u}^{\prime}-\kappa_{2} \nabla_{t} \cdot \mathbf{u}_{1}^{\prime},
$$

where $C_{u}^{\prime}$ is the local linearized curvature of the flame front, $\nabla_{t}$ is the divergence operator associated with the flame surface, and $\kappa_{1}$ and $\kappa_{2}$ are the Markstein lengths associated, respectively, with the curvature of the flame and with the local strain of the upstream flow. For a given input, this equation allows for the computation of the local value of $\xi$ and its action on the upstream flow.

The vorticity sheet is accounted for by the Biot-Savart law using conventions from Fig. 17 according to

$$
\mathbf{u}_{B S}^{\prime}(\mathbf{x})=\frac{1}{4 \pi} \int_{\mathrm{S}} \frac{\left(\mathbf{x}-\mathbf{x}^{\prime}\right)}{\left|\mathbf{x}-\mathbf{x}^{\prime}\right|^{3}} \times \mathbf{n}_{0}\left(\frac{\partial \xi}{\partial s}\left(v_{0,2}-v_{0,1}\right)\right) \mathrm{d} \mathrm{S}\left(\mathbf{x}^{\prime}\right),
$$

where $S$ denotes the flame front. This self-induced vorticity has been superimposed on the acoustic forcing for the computation of Eq. (37). The forcing has been taken as a periodic acoustic wave with a fixed frequency; a vorticity sheet is generated on the wall at the injection plane. This correction models the anchor-point dynamics and provides a Neumann boundary condition for Eq. (37),

$$
\omega_{\text {in }}=\frac{\partial \xi}{\partial s}\left(v_{0,2}-v_{0,1}\right),
$$

where $\omega_{\text {in }}$ is the input-speed discontinuity at the flame anchor-point. The importance of pipe corners in vorticity generation can be appreciated by an analysis of the Direct Navier-Stokes Simulation 
(DNS) results presented in the Appendix. Eqs. (37), (38), and (39) then form a closed set of linear equations.

The flame base-flow position has been set as the isolevel $\rho_{0}=0.7 \rho_{\infty}$. This value has been found to reasonably well interpolate the linear vorticity layer in the flame. The flame speed has been inferred from the two-dimensional results. For a given density level set, it has been extrapolated using

$$
S_{d, 0}=\left(\mathbf{u}_{0,2 D} \cdot \mathbf{n}_{0}\right) \frac{S_{r e f}}{S},
$$

where $S$ is the surface of the considered isolevel, and $S_{\text {ref }}$ is the surface of the level $\rho_{0}=0.99$, defined as the start of the preheating zone. This technique has been found to give consistent results for different density isolevels. The interpolated convective speed $u_{0}$ of Eq. (37) has been straightforwardly obtained with

$$
u_{0}=\mathbf{u}_{0,2 D} \cdot \mathbf{t}_{0} .
$$

The computation of the Markstein lengths has proven more challenging and has resulted in strong variations in the values estimated from base-flow analysis. The lengths were, respectively, set to $\kappa_{1}=0.05$ and $\kappa_{2}=0.005$. They are in qualitative agreement with observations from two-dimensional simulations. The direct implementation of a Biot-Savart law, like Eq. (38), is known to yield numerical instabilities and requires a desingularization $\mathrm{step}^{81}$ to produce a stable algorithm. The characteristic thickness of the sheet has been set to $1 / 3$ of the flame thickness.

The last challenge in the implementation of this test case concerns the setup of the acoustic forcing. In fully compressible reactive simulations, acoustic waves have been found to be reflected by the internal and external flame front because the flame acts as a wave guide. In a first approximation, this effect has been neglected. The forcing amplitude has been determined from the initialization of a linear acoustic forcing at $S t=2.64$. The phase has then been computed based on a delay model, assuming an acoustic wave propagating along the pipe axis, i.e.,

$$
\varphi=\mathrm{St}\left(t-\frac{x}{\mathrm{Ma}}\right) \text {. }
$$

Finally, the input vorticity $\omega_{\text {in }}$ has been calculated from the discontinuity of the axial velocity at wall corners. These techniques have not been able to predict the acoustic fringes observed in Fig. 14 and can only provide an estimate for the anchor-point dynamics. They are nonetheless intended as an illustration of the effect of vorticity on the upstream flow induced by flame deformations.

As the system is linear and forced at a chosen nondimensional frequency $S t$, the solution can be decomposed as

$$
\xi(s, t)=\hat{\xi}(s) \exp (i \text { St } t) .
$$

The equation for $\hat{\xi}$ is thus time-independent and has been solved using a preconditioned, iterative Generalized Minimal Residual Method (GMRES) algorithm. ${ }^{82}$

Results have been post-processed using the same methodology employed for Fig. 14. Both figures use the same colormap. As can be seen in Fig. 18, the model reproduces the first low-frequency region observed in Fig. 14, in blue, in the phase diagram. The amplitude of the velocity in this region is nearly constant, except for $S t=1.4$, for which a quick decay is observed. This effect is likely due to the definition of $T F_{F I O M}$ in Eq. (30) and associated with a spuriously high amplitude of $\tilde{u}(\tilde{s}=0, S t=1.4)$. The critical Strouhal number has been shifted from 6.3 to 3.6.

The structure of the transitional region has been found to strongly depend on $\kappa_{1}$ and $\kappa_{2}$ and on the values of the critical Strouhal number to be closely related to the anchor-point boundary conditions. However, this region extends from $S t=5$ to $S t=10$; furthermore, it is qualitatively correctly identified in frequency. For $S t>10$, as in Fig. 14, the acoustics dominate.

Given the simplicity of our approach, these results are very encouraging and highlight the need for a careful modeling of forcing terms and flame parameters when using a $G$-equation to represent the flame front. Additionally, the model shows the importance of coupling any nonlinear $G$-equation with a resolved continuity equation, in order to take into account the effects of the flame front displacement on the upstream flow. 


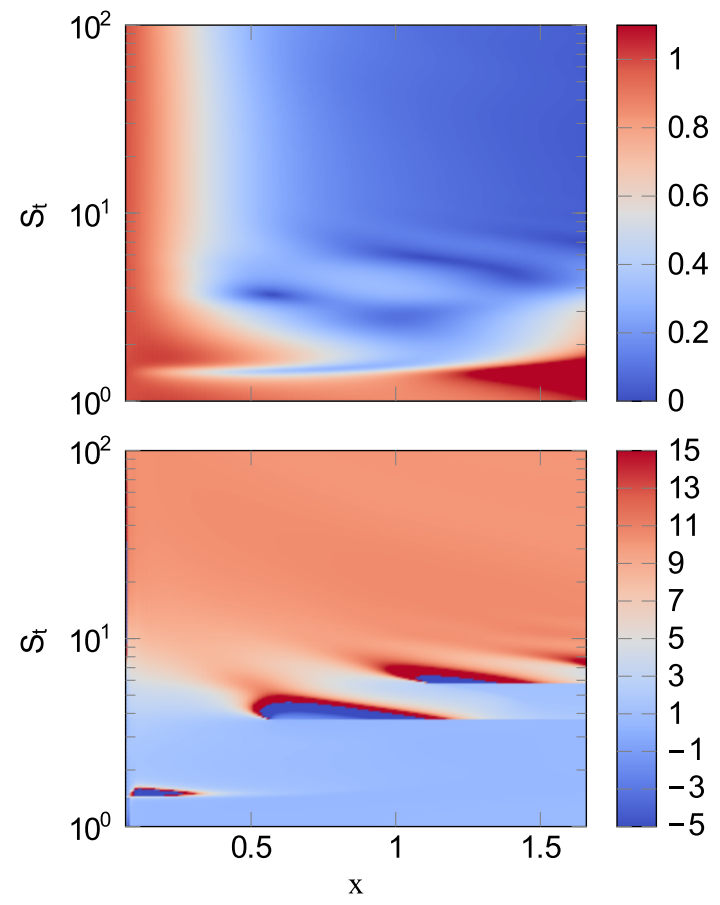

FIG. 18. Norm (top) and phase speed (bottom) of the $\mathrm{TF}_{F I O M}$ computed from the model.

Finally, this study demonstrated the efficiency of our linearization technique to analyze a physical process as complex as the one occurring in a flame. It enabled us to develop a model that has been found to correctly account for the bulk of the observed physical mechanisms.

\section{CONCLUSION}

Even though it is widely acknowledged that flames react sensitively to acoustic excitation, a frequency response analysis of flames to sources of sound and its dependence on governing parameters appears to be lacking. The present study is an attempt to propose a numerical approach based on linearized governing equations that provides insight into acoustically forced flame dynamics and delineates the role of hydrodynamics and reaction chemistry. To this end, the compressible Navier-Stokes equations, augmented by equations for species mass fraction and closed by a one-way, one-step chemistry model of Arrhenius type, have been linearized about an M-flame and used as a basis for a numerical frequency response analysis. The resulting flame transfer function has been found in good agreement with experimental data for a laminar premixed methane-air flame. The accuracy of the model over the considered frequency range is further corroborated by matching the heat-release fluctuations and sound emission to results from combustion noise theory.

The principal advantage of the numerical frequency response approach lies in its componentwise analysis of physical effects on a specific output quantity. In this manner, the role and dominance of purely hydrodynamic versus reactive processes can be determined and quantified independently. Furthermore, a pointwise input-output map is able to isolate regions in space that respond favorably and maximally to acoustic excitation at a given frequency.

This type of analysis, applied to the M-flame, confirmed the role of flame-front deformations in the production of hydrodynamic structures which dominate and co-exist with the acoustic field over a certain range of Strouhal numbers. In particular, acoustically induced vorticity at the flame front is purely hydrodynamically driven for $S t<6.3$. For higher Strouhal numbers, the convective mode and the acoustic wave co-exist, before the acoustic wave prevails for Strouhal numbers above 32. In the low-Strouhal regime, the convective mode is induced by a varicose response of the internal and external flame front to the acoustic forcing and dominates the flame front dynamics. A linearized 
model based on the $G$-equation has been developed to provide additional insight into the flame dynamics in the convection-dominated Strouhal-number regime. In this approach, the wrinkled flame sheet is modeled by a vorticity sheet which is driven by two linearized source terms capturing the effects of localized curvature and local strain. Supplemented by a Biot-Savart equation and an anchor-point boundary condition, this model has been able to qualitatively reproduce the observations of the frequency response analysis and to duplicate (even quantitatively) the regime-switching of the convective and acoustic modes as a function of the Strouhal number. The low-frequency response of the wrinkled M-flame is thus influenced by a feedback mechanism involving the convective mode which drives the dynamics of the flame tip and thus the majority of radiated noise.

\section{ACKNOWLEDGMENTS}

We wish to acknowledge the computational resources of GENCI-CINES and TGCC (Grant 2014-2a6451).

\section{APPENDIX: VOLUME VORTICITY SOURCES}

In this section, all linear simulations take into account unsteady chemistry terms. Our analytical model relies on the assumption of an infinitely thin flame. However, in DNS computations, the flame has a finite thickness. This section intends to provide information on vorticity generation inside the finite-thickness flame front.

Only a small amount of vorticity is present in the reaction layer of the base flow, as can be seen in Fig. 19. The vorticity of the base flow is mostly associated with the acceleration of the flow between the internal and external flame fronts. The volume vorticity observed in linear simulations thus cannot be explained by a convection of base-flow vorticity. This linear vorticity field associated with an acoustic forcing at $S t=2.64$ is presented in Fig. 20. Identifying the main vorticity sources is also of interest.

The vorticity equation for compressible flow can be expressed as

$$
\begin{aligned}
\frac{\partial \omega}{\partial t}+(\mathbf{u} \nabla) \omega & =(\omega \nabla) \mathbf{u}-\omega(\nabla \cdot \mathbf{u})+ \\
\frac{1}{\rho^{2}} \nabla \rho & \wedge \nabla p+\nabla \wedge\left(\frac{1}{\rho} \nabla \cdot \underline{\mathbf{\tau}}\right)+\nabla \wedge \mathbf{F},
\end{aligned}
$$

where $\omega$ is the vorticity, and $\mathbf{F}$ stands for volume forces. In our case, gravity is neglected and $\mathbf{F}=0$. Within an axisymmetric framework, it is convenient to group the first two terms of the right hand side; in the following, this first group will be referred to as the dilatation sources. The third term contains baroclinic vorticity sources, while the fourth captures viscous effects. A linearization of Eq. (A1) about a steady state thus provides expressions for the different components of the linear vorticity equation. These terms have been separately evaluated and are presented in Fig. 21.

Three distinct regions can be distinguished: (i) the flame tip, (ii) the quasi-planar flame-front region, comprising the internal and external flame fronts, and (iii) the anchor-points area. The

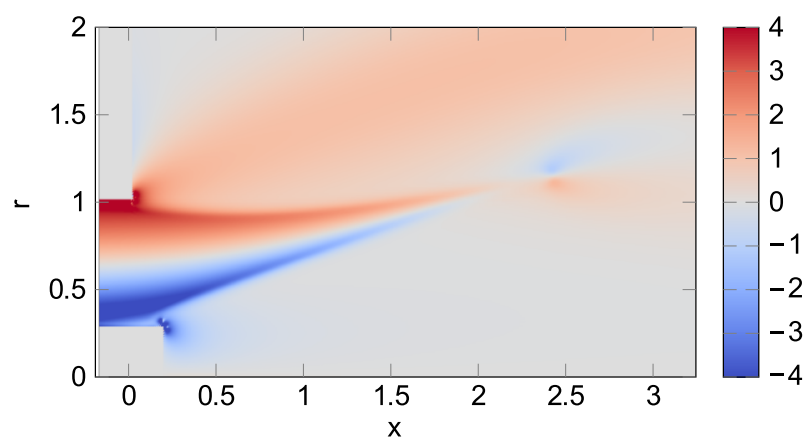

FIG. 19. Base-flow vorticity. 


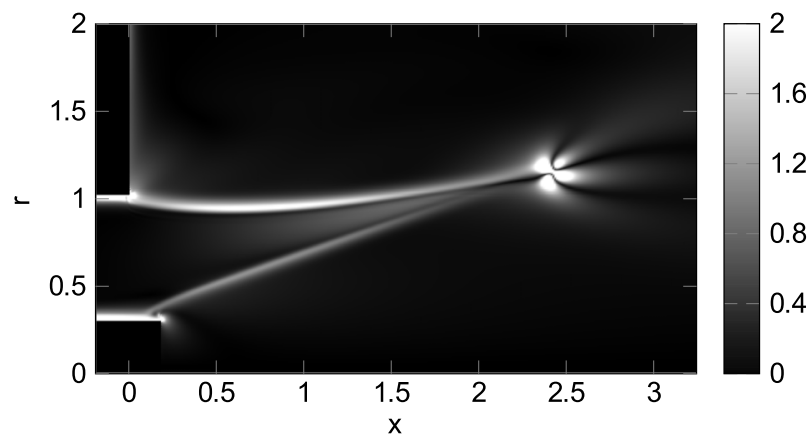

FIG. 20. Absolute value of the permanent-regime linear vorticity, with an acoustic forcing at $S t=2.64$.
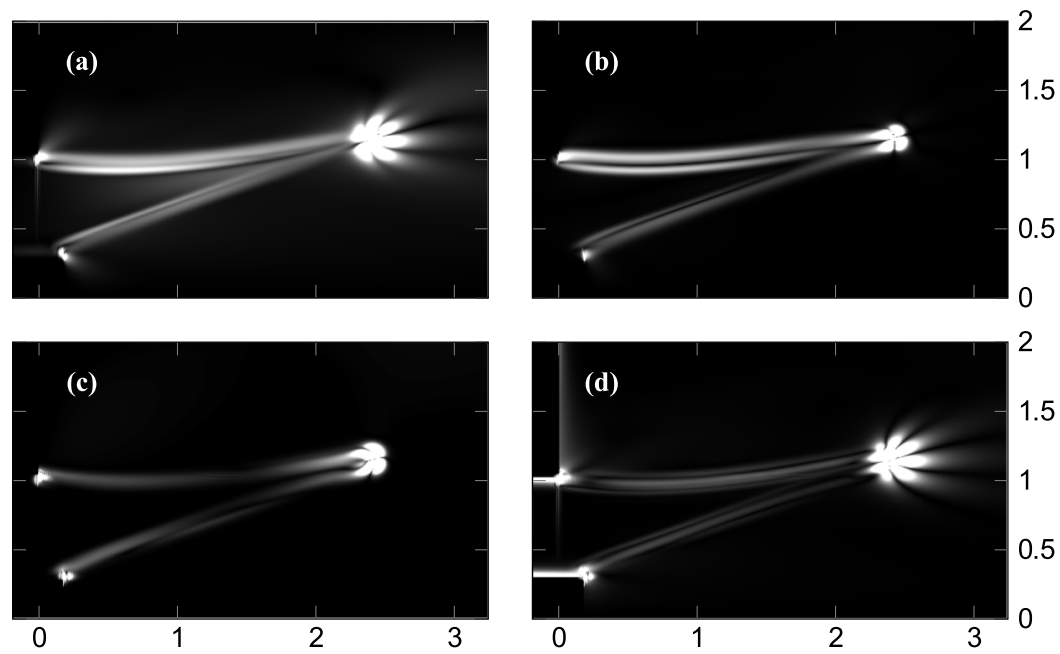

FIG. 21. Absolute value of the components of the vorticity sources: (a) vorticity convection sources; (b) dilatation sources; (c) baroclinic sources; (d) viscous sources. The colormap is the same for all figures.

vorticity at the flame tip can straightforwardly be interpreted as the consequence of the local, high heat release rate. The strong dilatation occurring in a restricted region leads to a strong, but localized vorticity field. In the quasi-planar flame front region, the dilatation counteracts the convection in the direction normal to the flame front. This causes confinement of the vorticity layer inside the flame front. The vorticity is only convected in the tangential direction. In this region, baroclinic and viscous effects are negligible. Finally, in the anchor-point area, baroclinic and viscous effects are dominant (by two orders of magnitude). Moreover, locally, viscous sources are one order of magnitude higher than baroclinic sources. This suggests that the vorticity is mostly generated at the anchor-points, inside the boundary layer, by viscous effects.

A simple mechanism can be suggested for this vorticity generation. The acoustic boundary layer is much thinner than the fluid-based boundary layer. At the exit, a strong radial gradient of axial velocity is thus induced at the corners. This shear generates vorticity through viscosity, which is subsequently convected along the flame front by the flow.

${ }^{1}$ S. Candel, D. Durox, S. Ducruix, A.-L. Birbaud, N. Noiray, and T. Schuller, "Flame dynamics and combustion noise: Progress and challenges," Int. J. Aeroacoust. 8(1), 1-56 (2009).

${ }^{2}$ Combustion Instabilities in Gas Turbines, Operational Experience, Fundamental Mechanisms, and Modeling, edited by

T. C. Lieuwen and V. Yang, Progress in Astronautics and Aeronautics Vol. 210 (American Institute of Aeronautics and Astronautics, Inc., 2005).

${ }^{3}$ T. C. Lieuwen, Unsteady Combustor Physics (Cambridge University Press, 2012).

${ }^{4}$ C. O. Paschereit, B. Schuermans, W. Polifke, and O. Mattson, "Measurement of transfer matrices and source terms of premixed flames," J. Eng. Gas Turbines Power 124, 239-247 (2002). 
${ }^{5}$ B. Schuermans, F. Guethe, D. Pennel, D. Guyot, and C. O. Paschereit, "Thermoacoustic modeling of a gas turbine using transfer functions measured at full engine pressure," J. Eng. Gas Turbines Power 132(11), 11503 (2010).

${ }^{6}$ D. Durox, T. Schuller, N. Noiray, and S. Candel, "Experimental analysis of nonlinear flame transfer functions for different flame geometries," Proc. Combust. Inst. 32(1), 1391-1398 (2009).

${ }^{7}$ K. T. Kim and S. Hochgreb, "The nonlinear heat release response of stratified lean-premixed flames to acoustic velocity oscillations," Combust. Flame 158, 2482-2499 (2011).

${ }^{8}$ K. T. Kim, J. G. Lee, B. D. Quay, and D. A. Santavicca, "Response of partially premixed flames to acoustic velocity and equivalence ratio perturbations," Combust. Flame 157, 1731-1744 (2010).

${ }^{9}$ B. Schuermans, F. Guethe, and W. Mohr, "Optical transfer function measurements for technically premixed flames," J. Eng. Gas Turbines Power 132, 081501 (2010).

${ }^{10}$ P. Palies, D. Durox, T. Schuller, and S. Candel, "Experimental study on the effect of swirler geometry and swirl number on flame describing functions," Combust. Sci. Technol. 183(7), 704-717 (2011).

${ }^{11}$ S. Schimek, J. P. Moeck, and C. O. Paschereit, "An experimental investigation of the nonlinear response of an atmospheric swirl-stabilized premixed flame,” J. Eng. Gas Turbines Power 133(10), 101502 (2011).

12 B. C. Bobusch, B. Cosic, J. P. Moeck, and C. O. Paschereit, "Optical measurement of local and global transfer functions for equivalence ratio fluctuations in a turbulent swirl flame," J. Eng. Gas Turbines Power 136, 021506 (2014).

${ }^{13}$ S. Bade, M. Wagner, C. Hirsch, T. Sattelmayer, and B. Schuermans, "Design for thermo-acoustic stability: Procedure and database," J. Eng. Gas Turbines Power 135, 121507 (2013).

${ }^{14}$ S. Bade, M. Wagner, C. Hirsch, T. Sattelmayer, and B. Schuermans, "Design for thermo-acoustic stability: Modeling of burner and flame dynamics," J. Eng. Gas Turbines Power 135, 111502 (2013).

${ }^{15}$ N. Noiray, D. Durox, T. Schuller, and S. Candel, "A unified framework for nonlinear combustion instability analysis based on the flame describing function,” J. Fluid Mech. 615, 139-167 (2008).

${ }^{16}$ P. Palies, D. Durox, T. Schuller, and S. Candel, "Nonlinear combustion instabilities analysis based on the flame describing function applied to turbulent premixed swirling flames," Combust. Flame 158, 1980-1991 (2011).

${ }^{17}$ W. Krebs, H. Krediet, E. Portillo, S. Hermeth, T. Poinsot, S. Schimek, and O. Paschereit, "Comparison of nonlinear to linear thermoacoustic stability analysis of a gas turbine combustion system," J. Eng. Gas Turbines Power 135, 081503 (2013).

${ }^{18}$ H. Krediet, C. H. Beck, W. Krebs, S. Schimek, C. O. Paschereit, and J. B. W. Kok, "Identification of the flame describing function of a premixed swirl flame from LES," Combust. Sci. Technol. 184, 888-900 (2012).

${ }^{19}$ L. Tay-Wo-Chong and W. Polifke, "Large eddy simulation-based study of the influence of thermal boundary condition and combustor confinement on premix flame transfer functions," J. Eng. Gas Turbines Power 135, 021502 (2013).

${ }^{20}$ S. Hermeth, G. Staffelbach, L. Y. M. Gicquel, V. Anisimov, C. Cirigliano, and T. Poinsot, "Bistable swirled flames and influence on flame transfer functions," Combust. Flame 161(1), 184-196 (2014).

${ }^{21}$ G. Staffelbach, L. Y. M. Gicquel, G. Boudier, and T. Poinsot, "Large eddy simulation of self excited azimuthal modes in annular combustors," Proc. Combust. Inst. 32, 2909-2916 (2009).

${ }^{22}$ P. Iudiciani and C. Duwig, "Large eddy simulation of the sensitivity of vortex breakdown and flame stabilisation to axial forcing," Flow, Turbul. Combust. 86, 639-666 (2011).

${ }^{23}$ B. Franzelli, E. Riber, L. Y. M. Gicquel, and T. Poinsot, "Large eddy simulation of combustion instabilities in a lean partially premixed swirled flame," Combust. Flame 159(2), 621-637 (2012).

${ }^{24}$ L. Tay-Wo-Chong, T. Komarek, R. Kaess, S. Foller, and W. Polifke, "Identification of flame transfer functions from LES of a premixed swirl burner," in Proceedings of ASME Turbo Expo 2010 (ASME, 2010), pp. 623-635.

${ }^{25}$ L. Tay-Wo-Chong, S. Bomberg, A. Ulhaq, and W. Polifke, "Comparative validation study on identification of premixed flame transfer function,” J. Eng. Gas Turbines Power 134(2), 021502 (2012).

${ }^{26}$ M. Fleifil, A. M. Annaswamy, Z. A. Ghoneim, and A. F. Ghoniem, "Response of a laminar premixed flame to flow oscillations: A kinematic model and thermoacoustic instability results," Combust. Flame 106, 487-510 (1996).

27 A. P. Dowling, "A kinematic model of a ducted flame," J. Fluid Mech. 394, 51-72 (1999).

${ }^{28}$ T. Schuller, D. Durox, and S. Candel, "A unified model for the prediction of laminar flame transfer functions: Comparisons between conical and V-flame dynamics," Combust. Flame 134(1-2), 21-34 (2003).

29 J. H. Cho and T. Lieuwen, "Laminar premixed flame response to equivalence ratio oscillations," Combust. Flame 140, $116-129$ (2005).

${ }^{30}$ Preetham, S. Hemchandra, and T. Lieuwen, "Dynamics of laminar premixed flames forced by harmonic velocity disturbances," J. Propul. Power 24(6), 1390-1402 (2008).

${ }^{31}$ H. M. Altay, S. Park, D. Wu, D. Wee, A. M. Annaswamy, and A. F. Ghoniem, "Modeling the dynamic response of a laminar perforated-plate stabilized flame," Proc. Combust. Inst. 32, 1359-1366 (2009).

32 P. Palies, T. Schuller, D. Durox, and S. Candel, "Modeling of swirling flames transfer functions," Proc. Combust. Inst. 33, 2967-2974 (2011).

${ }^{33}$ K. I. Matveev and F. E. C. Culick, "A model for combustion instability involving vortex shedding," Combust. Sci. Technol. 175, 1059-1083 (2003).

${ }^{34}$ A. L. Birbaud, D. Durox, and S. Candel, "Upstream flow dynamics of a laminar premixed conical flame submitted to acoustic modulations," Combust. Flame 146(3), 541-552 (2006).

${ }^{35}$ A. L. Birbaud, D. Durox, S. Ducruix, and S. Candel, "Dynamics of free jets submitted to upstream acoustic modulations," Phys. Fluids 19(1), 013602 (2007).

${ }^{36}$ P. Palies, T. Schuller, D. Durox, L. Y. M. Gicquel, and S. Candel, "Acoustically perturbed turbulent premixed swirling flames," Phys. Fluids 23, 037101 (2011).

37 V. Acharya, D.-H. Shin, and T. Lieuwen, "Swirl effects on harmonically excited, premixed flame kinematics," Combust. Flame 159(3), 1139-1150 (2012).

${ }^{38}$ C. W. Rhee, L. Talbot, and J. A. Sethian, “Dynamical behaviour of a premixed turbulent open V-flame," J. Fluid Mech. 300, 87-115 (1995).

${ }^{39}$ D. Hartmann, M. Meinke, and W. Schröder, "A level-set based adaptive-grid method for premixed combustion," Combust. Flame 158, 1318-1339 (2011). 
${ }^{40} \mathrm{~S}$. Hemchandra, "Premixed flame response to equivalence ratio fluctuations: Comparison between reduced order modeling and detailed computations," Combust. Flame 159, 3530-3543 (2012).

${ }^{41}$ K. Kashinath, S. Hemchandra, and M. Juniper, "Nonlinear thermoacoustics of ducted premixed flames: The influence of perturbation convection speed," Combust. Flame 160, 2856-2865 (2013).

42 V. N. Kornilov, R. Rook, J. H. M. ten Thije Bookkamp, and L. P. H. de Goey, "Experimental and numerical investigation of the acoustic response of multi-slit bunsen burners," Combust. Flame 156(10), 1957-1970 (2009).

${ }^{43}$ P. Auzillon, B. Fiorina, R. Vicquelin, N. Darabiha, O. Gicquel, and D. Veynante, "Modeling chemical flame structure and combustion dynamics in LES,” Proc. Combust. Inst. 33(1), 1331-1338 (2011).

${ }^{44}$ F. Duchaine, F. Boudy, D. Durox, and T. Poinsot, "Sensitivity of flame transfer functions of laminar flames," Combust. Flame 158, 2384-2394 (2011).

${ }^{45}$ K. S. Kedia, H. M. Altay, and A. F. Ghoniem, "Impact of flame-wall interaction on premixed flame dynamics and transfer function characteristics," Proc. Combust. Inst. 33, 1113-1120 (2011).

${ }^{46}$ X. Garnaud, "Modes, transient dynamics and forced response of circular jets," Ph.D. thesis (Ecole Polytechnique X, 2012).

${ }^{47}$ M. Lemke, J. Reiss, and J. Sesterhenn, "Adjoint based optimisation of reactive compressible flows," Combust. Flame 161(10), 2552-2564 (2014).

${ }^{48}$ U. A. Qadri, "Global stability and control of swirling jets and flames," PhD thesis (University of Cambridge, 2014).

${ }^{49}$ T. Ph. Bui, W. Schröder, and M. Meinke, "Numerical analysis of the acoustic field of reacting flows via acoustic perturbation equations," Comput. Fluids 37(9), 1157-1169 (2008).

${ }^{50}$ A. R. Kerstein, W. T. Ashurst, and F. A. Williams, "Field equation for interface propagation in an unsteady homogeneous flow field," Phys. Rev. A 37(7), 2728-2731 (1988).

${ }^{51}$ N. Peters, Turbulent Combustion (Cambridge University Press, 2000).

${ }^{52}$ S. Ducruix, T. Schuller, D. Durox, and S. Candel, "Combustion dynamics and instabilities: Elementary coupling and driving mechanisms,” J. Propul. Power 19(5), 722-734 (2003).

${ }^{53}$ L. Boyer and J. Quinard, "On the dynamics of anchored flames," Combust. Flame 82(1), 51-65 (1990).

${ }^{54}$ I. B. Zeldovich, G. I. Barenblatt, V. B. Librovich, and G. M. Makhviladze, Mathematical Theory of Combustion and Explosions (Consultants Bureau, New York, NY, 1985).

55 V. V. Bychkov and M. A. Liberman, "Dynamics and stability of premixed flames," Phys. Rep. 325(4-5), 115-237 (2000).

${ }^{56}$ P. Pelce and P. Clavin, "Influence of hydrodynamics and diffusion upon the stability limits of laminar premixed flames," J. Fluid Mech. 124, 219-237 (1982).

${ }^{57}$ G. I. Sivashinsky, "Nonlinear analysis of hydrodynamic instability in laminar flames I. Derivation of basic equations," Acta Astronaut. 4(11-12), 1177-1206 (1977).

${ }^{58}$ K.-L. Pan and R. Fursenko, "Characteristics of cylindrical flame acceleration in outward expansion," Phys. Fluids 20(9), 094107 (2008).

${ }^{59}$ V. N. Kornilov, K. R. A. M. Schreel, and L. P. H. de Goey, "Experimental assessment of the acoustic response of laminar premixed bunsen flames," Proc. Combust. Inst. 31, 1239-1246 (2007).

${ }^{60}$ F. Baillot, D. Durox, and R. Prud'Homme, "Experimental and theoretical study of a premixed vibrating flame," Combust. Flame 88, 149-168 (1992).

${ }^{61}$ A. L. Birbaud, S. Ducruix, D. Durox, and S. Candel, "The nonlinear response of inverted V-flames to equivalence ratio nonuniformities," Combust. Flame 154(3), 356-367 (2008).

${ }^{62}$ T. Schuller, D. Durox, and S. Candel, "Self-induced combustion oscillations of laminar premixed flames stabilized on annular burners," Combust. Flame 135(4), 525-537 (2003).

63 T. Poinsot and D. Veynante, Theoretical and Numerical Combustion, 3rd ed. (R.T. Edwards, Inc., 2012).

${ }^{64}$ R. D. Sandberg, "Governing equations for a new compressible Navier-Stokes solver in general cylindrical coordinates," Monograph No. AFM-07/07, School of Engineering Sciences, University of Southampton, 2007.

${ }^{65}$ R. Courant, K. Friedrichs, and H. Lewy, "On the partial difference equations of mathematical physics," IBM J. Res. Dev. 11(2), 215-234 (1967).

${ }^{66} \mathrm{~J}$. Berland, C. Bogey, O. Marsden, and C. Bailly, "High-order, low dispersive and low dissipative explicit schemes for multiple-scale and boundary problems," J. Comput. Phys. 224(2), 637-662 (2007).

${ }^{67}$ S. Gottlieb, "On high order strong stability preserving Runge-Kutta and multi step time discretizations," J. Sci. Comput. 25(1), 105-128 (2005).

${ }^{68}$ D. I. Ketcheson, "Highly efficient strong stability-preserving Runge-Kutta methods with low-storage implementations," SIAM J. Sci. Comput. 30(4), 2113-2136 (2008).

${ }^{69}$ S. Balay, J. Brown, K. Buschelman, V. Eijkhout, W. D. Gropp, D. Kaushik, M. G. Knepley, L. C. McInnes, B. F. Smith, and H. Zhang, "PETSc users manual," Technical Report ANL-95/11 - Revision 3.5, Argonne National Laboratory, 2013.

${ }^{70}$ S. Balay, W. D. Gropp, L. C. McInnes, and B. F. Smith, "Efficient management of parallelism in object oriented numerical software libraries," in Modern Software Tools in Scientific Computing, edited by E. Arge, A. M. Bruaset, and H. P Langtangen (Birkhäuser Press, 1997), pp. 163-202.

${ }^{71}$ P. Huerre and P. A. Monkewitz, "Absolute and convective instabilities in free shear layers," J. Fluid Mech. 159, 151-168 (1985).

72 A. Michalke, "Survey on jet instability theory," Prog. Aerosp. Sci. 21, 159-199 (1984).

${ }^{73}$ P. A. Monkewitz and K. Sohn, "Absolute instability in hot jets," AIAA J. 26(8), 911-916 (1988).

${ }^{74}$ L. Lesshafft, P. Huerre, P. Sagaut, and M. Terracol, "Nonlinear global modes in hot jets," J. Fluid Mech. 554, 393-409 (2006).

${ }^{75}$ E. Akervik, L. Brandt, D. S. Henningson, J. Hoepffner, O. Marxen, and P. Schlatter, "Steady solutions of the Navier-Stokes equations by selective frequency damping," Phys. Fluids 18(6), 068102-068106 (2006).

${ }^{76}$ M. F. de Pando, D. Sipp, and P. J. Schmid, "Efficient evaluation of the direct and adjoint linearized dynamics from compressible flow solvers," J. Comput. Phys. 231(23), 7739-7755 (2012).

77 P. Clavin and E. D. Siggia, "Turbulent premixed flames and sound generation," Combust. Sci. Technol. 78(1-3), 147-155 (1991). 
${ }^{78}$ M. Talei, M. J. Brear, and E. R. Hawkes, “A parametric study of sound generation by premixed laminar flame annihilation," Combust. Flame 159(2), 757-769 (2012).

${ }^{79}$ G. H. Markstein, Nonsteady Flame Propagation (Pergamon, 1964).

${ }^{80}$ J.-M. Truffaut, "Étude expérimentale de l'origine du bruit émis par les flammes de chalumeaux," Ph.D. thesis (Université de Provence-Aix-Marseille I, 1998).

${ }^{81}$ R. Krasny, "Desingularization of periodic vortex sheet roll-up,” J. Comput. Phys. 65(2), 292-313 (1986).

82 Y. Saad and M. Schultz, "GMRES: A generalized minimal residual algorithm for solving nonsymmetric linear systems," SIAM J. Sci. Stat. Comput. 7(3), 856-869 (1986). 